\title{
Modeling non-confined coronal flares: Dynamics and X-ray diagnostics
}

\author{
F. Reale ${ }^{1}$, F. Bocchino ${ }^{2}$, and G. Peres ${ }^{1}$ \\ 1 Dipartimento di Scienze Fisiche \& Astronomiche, Sezione di Astronomia, Università di Palermo, \\ Piazza del Parlamento 1, 90134 Palermo, Italy \\ e-mail: peres@astropa.unipa.it \\ 2 Osservatorio Astronomico di Palermo G.S. Vaiana, Piazza del Parlamento 1, 90134 Palermo, Italy \\ e-mail: bocchino@stropa.unipa.it
}

Received 24 July 2001 / Accepted 11 December 2001

\begin{abstract}
Long-lasting, intense, stellar X-ray flares may approach conditions of breaking magnetic confinement and evolving in open space. In the perspective of searching for possible tracers of non-confinement, we explore this hypothesis with hydrodynamic simulations of flares occurring in a non-confined corona: model flares are triggered by a transient impulsive heating injected in a plane-parallel stratified corona. The plasma evolution is described by means of a numerical 2-D model in cylindrical geometry $R, Z$. We explore the space of fundamental parameters. As a reference model, we consider a flare triggered by a heating pulse of $10 \mathrm{erg} \mathrm{cm}^{-3} \mathrm{~s}^{-1}$ lasting $150 \mathrm{~s}$ and released in a region $\sim 10^{9} \mathrm{~cm}$ wide and at a height $\sim 2 \times 10^{9} \mathrm{~cm}$ from the base of the stellar surface. The pressure at the base of the corona of the unperturbed atmosphere is 0.1 dyne $\mathrm{cm}^{-2}$. The heating would cause a $20 \mathrm{MK}$ flare if delivered in a $40000 \mathrm{~km}$ long closed loop. The modeled plasma evolution in the heating phase involves the propagation of a $10 \mathrm{MK}$ conduction front and the evaporation of a shocked bow density front upwards from the chromosphere. As the heating is switched off, the temperature drops in few seconds while the density front still propagates, expanding, and gradually weakening. This kind of evolution is shared by other simulations with different coronal initial pressure, and location, duration and intensity of the heating. The X-ray emission, spectra and light curves at the ASCA/SIS focal plan, and in two intense X-ray lines (Mg XI at 9.169 $\AA$ and Fe XXI at $128.752 \AA$ ), have been synthesized from the models. The results are discussed and compared to features of confined events, and scaling laws are derived. The light curves invariably show a very rapid rise, a constant phase as long as the constant heating is on, and then a very fast decay, on time scales of few seconds, followed by a more gradual one (few minutes). We show that this evolution of the emission, and especially the fast decay, together with other potentially observable effects, are intrinsic to the assumption of non-confinement. Their lack indicates that observed long-lasting stellar X-ray flares should involve plasma strongly confined by magnetic fields.
\end{abstract}

Key words. stars: flare - stars: coronae - X-rays: stars - hydrodynamics

\section{Introduction}

Stellar coronae are not spatially resolved with present day observations. It is widely accepted that they share many of the basic mechanisms of the solar corona, the only resolved corona that we know. Decades of X-ray observations have shown that both its structuring and heating are dominated by the ambient magnetic field. Evidence has been found, such as the presence of coronal plasma at temperatures of several million degrees (e.g. Linsky 1981, Schmitt et al. 1990), that some form of confinement of Xray bright plasma should be at work on stars, probably associated with intense magnetic fields. It is then natural to wonder what is the morphology of the confining structures in a stellar corona. On the Sun, plasma is typically

Send offprint requests to: F. Reale,

e-mail: reale@astropa.unipa.it confined in magnetic arches (the loops), and both the geometry and luminosity of many of them are steady for relatively long times.

If stellar coronae are similar to the solar one, then structures similar to solar loops should be found on stars. Several approaches are possible to determine the dimensions of coronal structures. An eclipse on Algol has allowed to find the volume responsible of a flare (Schmitt \& Favata 1999). Loop fitting has been used to constrain the average linear dimensions of stellar coronal loops (e.g. Maggio \& Peres 1997). Another source of information on the characteristic of stellar coronal loops is the evolution of X-ray flares. In particular, basic mechanisms of plasma cooling are radiation and thermal conduction from the corona downwards to the cooler chromosphere. Linking the plasma characteristic cooling times to the observed flare decay time allows to infer the linear size of the 
cooling region. This property has been largely exploited in the past to estimate the size of stellar flaring loops (e.g. Haisch 1983; van den Oord et al. 1988; van den Oord \& Mewe 1989; Reale et al. 1988; Reale et al. 1997; Favata \& Schmitt 1999; see Reale 2001 for a review).

One implication of the simple scaling from cooling times is that slower and slower decays, associated with long-lasting flares, correspond to longer and longer loops. As a matter of fact, stellar X-ray flares are typically observed to last much longer than average solar X-ray flares, up to several days whereas the duration of solar flares ranges from few minutes to several hours. In spite of the obvious observational bias toward detecting long events, the evidence of long flares has been taken as strong indication of very large flaring regions, in particular comparable to, or even larger than, the stellar radius (e.g. Graffagnino et al. 1995; Favata \& Schmitt 1999; Tsuboi et al. 2000; Favata 2001). On the Sun, large scale and long-duration events typically occur in complex coronal structures, in which the magnetic field undergoes major rearrangements and several loop structures are progressively involved (e.g. two-ribbon flares): the large amounts of energy released lead to large plasma pressure which may exceed the magnetic one and cause the end of confinement. The question now is: may plasma in long-lasting and intense stellar flares break the magnetic cage, and erupting, be free to move and evolve in open space?

This work addresses this question, by modeling flares occurring in a totally non-confined stratified corona. Although we do not pretend this to be an entirely realistic scenario, it can be considered as an extreme limit of situations in which the plasma dynamics dominates over the confining magnetic field and therefore governs the X-ray luminosity evolution.

Indeed, some solar and stellar flares show phenomena that indicate that at least some of the plasma is not confined. In the solar case, coronal mass ejections (CMEs) following flares are not confined. In the stellar case VLBI measurements of nonthermal particles propagating away from the star are observed. A good example is a flare in UX Ari observed by Mutel et al. (1985). White and Franciosini (1995) also show that the relativistic plasma responsible for the gyrosynchrotron radio emission in stellar comes from expanding plasma.

Here we focus on studying the X-ray emitting plasma. Our scope is to explore: to what point actual stellar flares can approximate free-expanding flaring plasma, if there are distinctive signatures in X-ray emission (obtained from the modeling) which characterize unconfined flares vs confined ones and therefore allow us to discriminate the confining role of the magnetic field, and if observed stellar flares present such signatures.

The study that we illustrate here presents also interesting results on the theoretical point of view: we describe the evolution of the non-confined plasma in a stratified coronal atmosphere, i.e. which is hot ab initio and where thermal conduction is fully efficient. This aspect makes this work quite different from typical models of bursts, like supernovae, which instead propagate in non-conducting and cooler media.

Our approach is to set up an initial stratified atmosphere, similar to a confined one, including chromosphere, transition region and corona, and where the plasma is not confined to move along one spatial direction, and then to impart a localized energy impulse. Plasma dynamics and heat conduction can occur isotropically. The evolution of such system needs to be described by a numerical 2-D hydrodynamic model, with enhanced spatial resolution in the low corona and in the transition region and including plasma thermal conduction; therefore it is also a demanding numerical problem. In order to compare our results with observations and to obtain diagnostical feedbacks, we synthesize from our model results the expected evolution of the integrated X-ray flare luminosity, i.e. by simulating a flaring unresolved source, such as a star. To have a realistic scenario, representative of real flare observations, we have chosen to synthesize the emission in a wide X-ray band such as at the focal plane of the ASCA/SIS (sensitive in the range, approximately, $0.3-10 \mathrm{keV}$ ), and in two intense X-ray lines. Similar results can be expected for other wide band CCD instruments such as Chandra/ACIS and $\mathrm{XMM} / \mathrm{EPIC}$, while the lines may be detectable by instruments with good spectral resolution such as Chandra and XMM-Newton grating detectors.

In Sect. 2 we describe our modeling approach in detail, in Sect. 3, the simulations performed and the results obtained are presented, in Sect. 4 the results are discussed and in Sect. 5 conclusions are drawn.

\section{Modeling}

\subsection{The model set up}

The general concept of our modeling non-confined coronal flares is similar to modeling confined flares (e.g. Peres et al. 1982): the flare is triggered by an impulsive heating injected in a stratified plane-parallel static corona. The basic difference is that the unperturbed atmosphere is not a semicircular coronal loop and that the heat transport, as well as any plasma motion, is allowed in any direction, instead of being limited only along magnetic field lines parallel to the loop flux tube. The evolution of a heat perturbation propagating in any direction in a stratified atmosphere can be properly described with a 2-D cylindrical geometry $(R, Z)$. This description is adequate for our purposes of deriving the global properties of the plasma evolution and X-ray emission as it would be detected at stellar distances.

The solar template of confined coronal flares has guided us in configuring the initial unperturbed atmosphere and in tuning the plasma and heating parameters. As shown in Fig. 1, the initial atmosphere consists of a stratified plane-parallel corona extending vertically from the stellar surface. The limit of our computational domain is at $10^{11} \mathrm{~cm}$ from the stellar surface. The corona is maintained steady at coronal conditions by a uniformly 


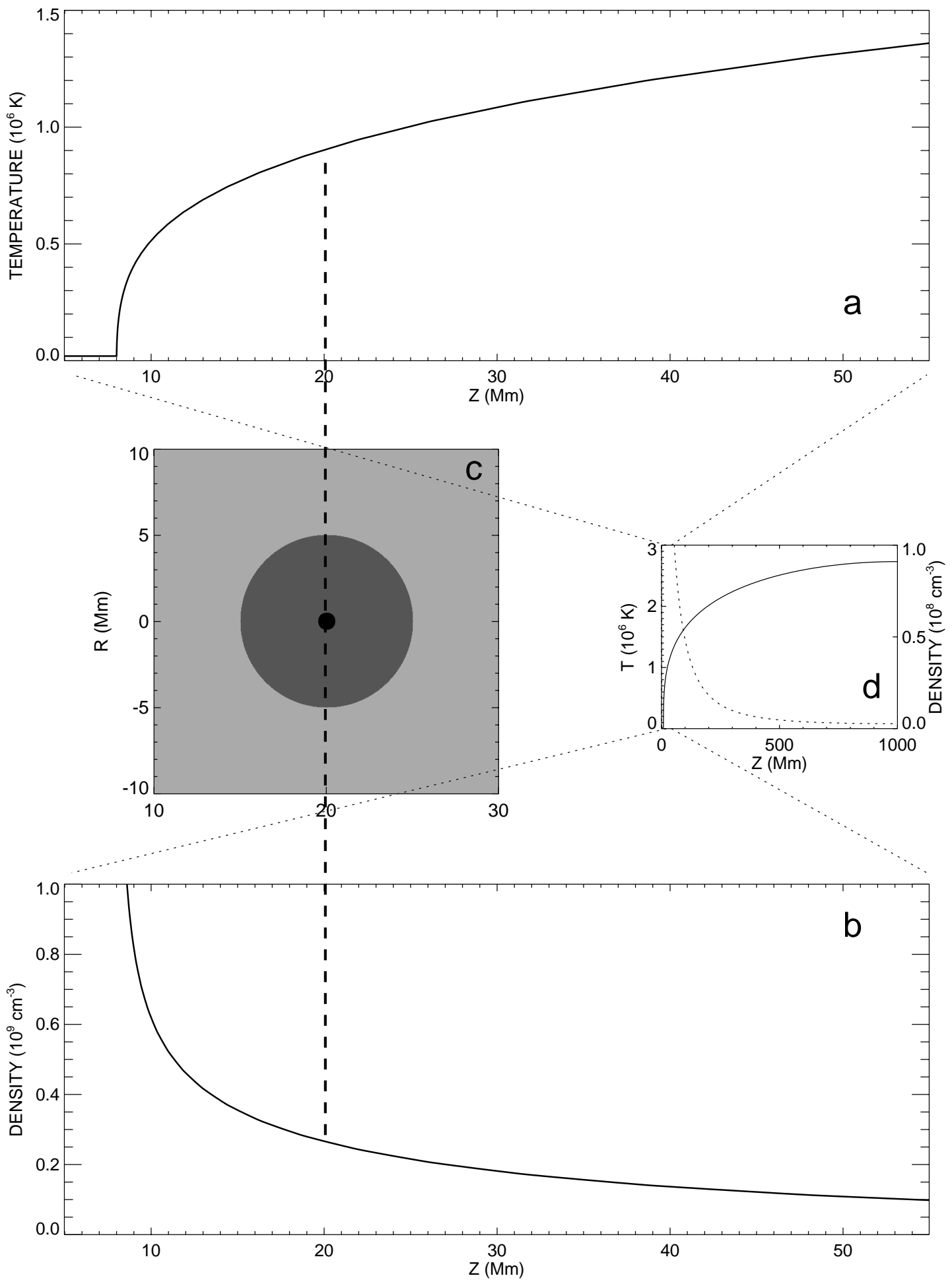

Fig. 1. Initial configuration of the reference model (cf. model 1 in Table 1); the geometry is cylindrical with coordinates $r, z$. Panels a) and b) show the temperature and density distributions along the vertical $z$ axis $(r=0)$. Panel c) shows the spatial distribution of the impulsive heating vs radial distance $(R)$ and height $(Z)$, where the radius of the dark grey circle is $\sigma_{\mathrm{H}}$ (see Eqs. (5), (6)). Notice that the upward vertical direction is rightwards in panel c). Panels a), b) and c) show a limited region of the whole computational domain. Panel $\mathbf{d}$ ) shows the temperature and density (dashed) distributions along the vertical $z$ direction on a much larger scale.

distributed heating. As our reference starting conditions we have taken an atmosphere with a pressure at the base of the corona of 0.1 dyne $\mathrm{cm}^{-2}$, typical of coronal quiet regions, which may reasonably approximate non-confined coronal conditions. In these conditions the temperature of the upper layers of the non-flaring corona of our 
computational domain is below $3 \mathrm{MK}$. We have made some simulations also with a base pressure 1 dyne $\mathrm{cm}^{-2}$, a denser and hotter $(\leq 6 \mathrm{MK})$ initial atmosphere, to check the effect of changing the initial conditions to rather extreme cases of very active "non-confined" coronae. The bottom of the corona is matched to a much cooler chromosphere through a steep transition region. All this setting is similar to that for modeling confined coronal flares (e.g. Peres et al. 1982; Cheng et al. 1983; McClymont \& Canfield 1983). The chromosphere is required as mass reservoir for the flare evolution; our choice has been to consider an isothermal $\left(2 \times 10^{4} \mathrm{~K}\right)$ and non-radiating chromosphere (Cheng et al. 1983). While the detailed structure of the chromosphere is important for the fine details of the evaporation and coronal evolution, a coarse chromospheric model like the one used here suffices to show the general characteristics of the evolution addressed here. We have verified that the unperturbed atmosphere is steady and stable for times much longer than the flare evolution times considered for this study.

The plasma evolution is described by solving the 2-D plasma hydrodynamic equations of conservation of mass, momentum and energy with the geometry described above:

$$
\begin{aligned}
& \frac{\partial n}{\partial t}+\nabla \cdot(n \boldsymbol{v})=0 \\
& \frac{\partial(n \boldsymbol{v})}{\partial t}+\nabla \cdot(n \boldsymbol{v} \boldsymbol{v})=-\frac{1}{m} \nabla p+n \boldsymbol{g} \\
& \frac{\partial u}{\partial t}+\nabla \cdot[(u+p) \boldsymbol{v}]=m n \boldsymbol{g} \cdot \boldsymbol{v}-n^{2} P(T)+\mathcal{H}(\boldsymbol{r})-\nabla F_{\mathrm{c}} \\
& u=\frac{1}{2} m n v^{2}+\mathcal{E}, p=(\gamma-1) \mathcal{E} \\
& T=\frac{p}{2 k n}, F_{\mathrm{c}}=-\kappa T^{5 / 2} \nabla T
\end{aligned}
$$

where $n$ particle density per unit volume, $t$ time, $\boldsymbol{v}$ the plasma bulk velocity, $p$ pressure, $m$ average mass per particle ( $m=2.1 \times 10^{-24} \mathrm{~g}$ for solar abundance), $\boldsymbol{g}$ gravity acceleration, $P(T)$ radiative losses per unit emission measure, $\mathcal{H}$ heating per unit volume (uniform and constant in transition region), $\mathcal{E}$ is the internal energy density, $T$ plasma temperature, $F_{\mathrm{c}}$ heat flux, $\kappa$ plasma thermal conductivity and $\gamma=5 / 3$. The flare is assumed to occur on a solar-like star, with solar surface gravity and radius.

Plasma thermal conduction is isotropic. Radiative losses are those of Raymond \& Smith (1977) but are set to zero for $T \approx 2 \times 10^{4} \mathrm{~K}$, i.e. for chromospheric plasma. The heating term generally consists of a steady heating term which maintains the unperturbed atmosphere in thermal equilibrium and of a transient term, which triggers the flare. In our simulations, the steady heating has been set to zero both in the chromosphere and elsewhere, since the transient heating is switched on, in order to describe the free plasma cooling with no interference from any heating source.
The transient heating term has been assumed as a separable function of space and time:

$H(Z, R, t)=H_{0} f(Z, R) g(t)$

The spatial distribution of the heating is a $2-\mathrm{D}$ circular Gaussian:

$f(Z, R)=\exp \left[\frac{\left(Z-Z_{\mathrm{H}}\right)^{2}+\left(R-R_{\mathrm{H}}\right)^{2}}{2 \sigma_{\mathrm{H}}^{2}}\right]$.

As for the temporal evolution $g(t)$ the impulsive heating has been assumed to be switched on at $t=0(g(0)=0)$, kept constant $(g(t)=1)$ for a given time lapse $0 \leq t \leq t_{\mathrm{H}}$ and then switched off $g\left(t>t_{\mathrm{H}}\right)=0$.

\subsection{The code}

The conservation equations shown above are solved numerically on a fixed geometrical grid. The time integration is made with "time splitting" in two separate phases: a) the proper hydrodynamic phase, which includes the mass and momentum equations and the advective terms of the energy equation; b) the thermal conduction phase, which includes also radiation and heating processes. The hydrodynamic section is based on an explicit Flux Corrected Transport (FCT) numerical scheme, improved for efficiency and accuracy in large-scale smooth regions (Reale et al. 1990). The highly non-linear thermal conduction is treated with an Alternating Direction Implicit (ADI) scheme (Reale 1995), which warrants unconditional stability. The two phases are solved independently according to a time-splitting approach, i.e. using different integration time steps, which depend on the characteristic times of the physical effects involved in each section and on the stability criteria of the respective numerical schemes, i.e. FCT and ADI.

As mentioned above the geometrical domain is 2-D in cylindrical coordinates $(R, Z)$. The grid does not change during a simulation and the grid cells are rectangular: the spacing $d Z$ along the $Z$ direction is uniform in the chromosphere $\left(d Z=2 \times 10^{6} \mathrm{~cm}\right)$ and then monotonically increases with a geometric progression up to $d Z \sim 2 \times 10^{9} \mathrm{~cm}$ at $Z \approx 10^{11} \mathrm{~cm}$; this spacing warrants a stable and steady atmosphere in equilibrium conditions; the spacing along $R$ is uniform as far as $7 \times 10^{10} \mathrm{~cm}$ from the central axis and then monotonically increases as far as $\sim 10^{11} \mathrm{~cm}$. The spacing along $Z$ and $R$ has been chosen so as to have a relatively high resolution and a regular grid in the internal domain region, where the energy is released and the flare mostly occurs, and at the same time, a very large domain (Bedogni \& Woodward 1990), so that the boundary conditions at the far extremes become unimportant for the problem under analysis. The space cells along $Z$ are 800 for all simulations performed. Along $R$ most simulations has been done with 200 grid cells for the internal uniform region, plus 28 cells of non-uniform expanding grid; some simulations have been done with 100 and 400 grid cells for the internal region. 
Reflecting boundary conditions have been set for the central $Z(R=0)$ axis. Density and temperature have been set constant on the lower $R$ axis $(Z=0)$, and with zero gradient on the upper one. The $Z$ component of the velocity is set to zero at both $Z$ boundaries, the $R$ component with zero gradient. At the far extreme of the $R$ domain, zero gradient conditions have been set for all the relevant quantities.

The code has been parallelized on the basis of a geometric domain decomposition and using the High Performance Fortran (HPF). In the Alternating Direction Implicit (ADI) scheme, the equation is integrated at each time step in two half steps. At each half-step the numerical integration advances in one of the two spatial directions. The integration scheme at each half-step is implicit: the solution at one grid-point is derived from the solution at the same time step at an adjacent point. The data dependencies prevent direct parallelization of the nested DO loops, as in the case of the FCT section: we have adopted a parallelization solution which minimizes the interprocessor communications and the sequential operations, without changing the numerical algorithm. Simulations have been executed on the Cray T3E at CINECA (Bologna, Italy), on a Compaq server and on a Compaq HPC Cluster at Osservatorio Astronomico di Palermo (Italy).

\section{The simulations}

\subsection{The parameter space and the reference flare}

Table 1 shows the parameters and some results of the simulations of non-confined flares presented here (plus one model of confined flare for comparison). For each simulation, there are the basic simulation parameters, i.e. the pressure at the base of the initial unperturbed corona, and the parameters of the heating release (see Eqs. (5) and (6)), i.e. the heating rate per unit volume $H_{0}$, the height $Z_{\mathrm{H}}$ of the heating maximum, the Gaussian width $\sigma_{\mathrm{H}}$, the total rate, and the duration of the heating (Eq. (5)), and some relevant results, i.e. the plasma maximum temperature, the maximum density at the position of the maximum temperature, the maximum velocity throughout the computed flare evolution, and, finally, the light curve decay time after the initial very fast decay phase (see Sect. 3.3).

Our simulation strategy is to set up and compute a reference model case and then perform other calculations by changing the parameters relevant for our study one by one. Our reference configuration has a coronal base pressure $p_{0}=0.1$ dyne $\mathrm{cm}^{-2}$, which is a value typical of quiet coronal regions or even of open coronal structures on the Sun. Since we suppose that higher pressures may occur on active stars, values up to $p_{0}=1$ dyne $\mathrm{cm}^{-2}$ have also been considered.

In our reference simulation the flare is triggered by a heating pulse with maximum rate rate per unit volume $H_{0}=10 \mathrm{erg} \mathrm{cm}^{-3} \mathrm{~s}^{-1}$ and width of the Gaussian $\sigma_{\mathrm{H}}=5 \times 10^{8} \mathrm{~cm}$ centered on the $Z$ axis $\left(R_{\mathrm{H}}=0\right)$ at a height of $Z_{\mathrm{H}}=2 \times 10^{9} \mathrm{~cm}$ above the base of the atmosphere, well above the transition region (Eq. (6)). Similar heating rate, width and position would, for instance, ignite a $20 \mathrm{MK}$ confined solar flare at the top of an active region loop (Peres et al. 1987), which may be taken for comparison to the results presented here. As shown in Table 1, other simulations have been performed with higher values of the rate $H_{0}$ up to $100 \mathrm{erg} \mathrm{cm}^{-3} \mathrm{~s}^{-1}$, in order to explore the trend toward more intense events such as those typical of active stars. We have also considered heat deposited higher in the corona, i.e. more distant from the stellar surface, and, at the same time, over a more extended volume, while either maintaining the same total heating rate or doubling it. In order to obtain a significant brightening over the background atmosphere, in all simulations with higher base coronal pressure, the heating rate $H_{0}$ is higher than the reference value, since the atmosphere is brighter and also more efficient in radiating the additional heat, this making any smaller heating barely effective.

For most simulations, the duration of the constant heating (the time in which $g(t)=1$ ) is $150 \mathrm{~s}$, but a case of much longer heating $\left(t_{\mathrm{H}}=600 \mathrm{~s}\right)$ has also been explored.

The reference simulation (named ref in Table 1) will be described in more detail in the following. With the total heating rate of $\sim 2 \times 10^{28} \mathrm{erg} \mathrm{s}^{-1}$, the total heat released is therefore $\sim 3 \times 10^{30} \mathrm{erg}$. The evolution is computed for a total time of $800 \mathrm{~s}$, i.e. the decay is studied for $650 \mathrm{~s}$. The grid is $800 \times 228$ grid cells.

For comparison with the models of flares in nonconfined atmospheres, Table 1 shows also the parameters and results of a model of flare in a confined coronal loop, labelled with Conf. In particular, the parameters are those of a flare in an active region loop of half-length $2 \times 10^{9} \mathrm{~cm}$ and initial pressure 6 dyne $\mathrm{cm}^{-2}$ (Peres et al. 1982; Peres et al. 1987). The heating duration, spatial extent and intensity per unit volume have been chosen identical to those of the reference model. A loop cross-section area of $2.5 \times 10^{17} \mathrm{~cm}^{2}$ has been assumed, corresponding to a loop aspect, i.e. radius divided by half-length, of 0.1 .

\subsection{The plasma evolution of the reference flare}

\subsubsection{The heating phase}

Figure 2 shows distributions of temperature, density, pressure and vertical component of velocity along the central $Z$ axis at various times during the phase in which the heating is switched on in the reference simulation. Figure 3 shows the analogous results of the evolution after the heating has been switched off. Figures 4 and 5 respectively show grey-scale images of the temperature and density contrasts $T / T_{0}$ and $n / n_{0}$, where $T_{0}$ and $n_{0}$ are the temperature and density of the unperturbed atmosphere at various times during the same simulation, including both heating and decay phase. Figures 2, 4 and 5 clearly show the evolution of the plasma in the heating phase.

Because of the strong impulsive heating the plasma temperature at the center of the heating release on the $Z$ 
Table 1. Parameters of simulated non-confined flares + one confined flare.

\begin{tabular}{lcccccccccc}
\hline Name & $\begin{array}{c}p_{0}^{a} \\
\mathrm{dyn} / \mathrm{cm}^{2}\end{array}$ & $\begin{array}{c}H_{0}^{b} \\
\mathrm{erg} \mathrm{cm}^{-3} \mathrm{~s}^{-1}\end{array}$ & $\begin{array}{c}Z_{\mathrm{H}}^{c} \\
10^{9} \mathrm{~cm}\end{array}$ & $\begin{array}{c}\sigma_{\mathrm{H}}^{d} \\
10^{9} \mathrm{~cm}\end{array}$ & $\begin{array}{c}H_{\mathrm{tot}}^{e} \\
10^{28} \mathrm{erg} / \mathrm{s}\end{array}$ & $\begin{array}{c}t_{\mathrm{H}}^{f} \\
\mathrm{~s}\end{array}$ & $\begin{array}{c}T_{\max }^{g} \\
10^{7} \mathrm{~K}\end{array}$ & $\begin{array}{c}D_{\max }^{h} \\
10^{9} \mathrm{~cm}^{-3}\end{array}$ & $\begin{array}{c}V_{\text {max }}^{i} \\
\mathrm{~km} \mathrm{~s}^{-1}\end{array}$ & $\begin{array}{c}\tau_{\mathrm{dec}}^{j} \\
\mathrm{~s}\end{array}$ \\
\hline Ref & 0.1 & 10 & 2.0 & 0.5 & 2 & 150 & 1.3 & 0.9 & 800 & 290 \\
Long & 0.1 & 10 & 2.0 & 0.5 & 2 & 600 & 1.3 & 0.9 & 1000 & 380 \\
H30 & 0.1 & 30 & 2.0 & 0.5 & 6 & 150 & 1.7 & 1.2 & 1000 & 270 \\
H100 & 0.1 & 100 & 2.0 & 0.5 & 20 & 150 & 2.5 & 1.9 & 1200 & 320 \\
X5H5 & 0.1 & 5 & 5.0 & 1.0 & 8 & 150 & 1.6 & 0.3 & 700 & 180 \\
X5H10 & 0.1 & 10 & 5.0 & 1.0 & 16 & 150 & 2.0 & 0.4 & 900 & 210 \\
P03H30 & 0.3 & 30 & 2.0 & 0.5 & 6 & 150 & 1.7 & 1.7 & 1000 & 350 \\
P03H100 & 0.3 & 100 & 2.0 & 0.5 & 20 & 150 & 2.5 & 2.6 & 800 & 370 \\
P1 & 1.0 & 100 & 2.0 & 0.5 & 20 & 150 & 1.9 & 2.8 & 600 & 200 \\
\hline Conf & 6 & 10 & 2.0 & 0.5 & 0.2 & 150 & 2.1 & 75 & 360 & $250^{l}$ \\
\hline
\end{tabular}

${ }^{a}$ Pressure at the base of the corona.

${ }^{b}$ Maximum heating rate per unit volume (see Eq. (5)).

${ }^{c}$ Height of the center of the heating (see Eq. (5)).

${ }^{d}$ Width of the heating (see Eq. (5)).

$e$ Total heating rate.

$f$ Heating duration (see Eq. (5)).

${ }^{g}$ Flare maximum temperature.

${ }^{h}$ Maximum density at the position of the temperature maximum.

${ }^{i}$ Maximum velocity.

${ }^{j}$ Light curve (in the ASCA/SIS band) $e$-folding decay time after the initial fast decay phase.

${ }^{k}$ Model of a flare confined in a solar active-region loop.

${ }^{l} E$-folding decay time since the light curve maximum.

axis increases rapidly (in less than $2 \mathrm{~s}$ ) from 1.5 to $12 \mathrm{MK}$ (and similarly the pressure by a factor 10) and then remains practically constant there. A thermal conduction front develops and propagates spherically and very rapidly in such a thin corona, taking $\sim 2 \mathrm{~s}$ to reach the base of the corona, the transition region and the chromosphere, and, on the opposite side, a height $Z \sim 5 \times 10^{9} \mathrm{~cm}$. As the conduction front propagates in corona, expanding from the heating region, it fades and slows down significantly. While a height $Z=10^{10} \mathrm{~cm}$ is reached by the temperature change after only $\sim 15 \mathrm{~s}$, it takes one minute more to reach $Z=2 \times 10^{10} \mathrm{~cm}$, and at the end of the heating the conduction front is only slightly higher than that. In the orthogonal (radial) direction the conduction front is slower, because the temperature increases upwards, and therefore so does the efficiency of thermal conduction, while this does not occur in the radial direction; $R=10^{10} \mathrm{~cm}$ is reached only after $50 \mathrm{~s}$ since the beginning of the simulation and at the end of the heating the distance of the conduction front from the $Z$ axis is $R=1.5 \times 10^{10} \mathrm{~cm}$. To have an idea of the weakening of the expanding conduction front, note that, at the end of the heating, the temperature is below $10 \mathrm{MK}$ farther than $2 \times 10^{9} \mathrm{~cm}$ from the center of the heating release and below 4 MK for $R>10^{10} \mathrm{~cm}$.

As soon as the chromosphere is hit and heated by the conduction front, it expands upwards with a shocked density front ${ }^{1}$. This is the chromospheric evaporation modeled in confined solar coronal flares (e.g. Nagai 1980; Peres et al. 1982). The plasma maximum speed of the evaporation front rapidly increases to $\sim 600 \mathrm{~km} \mathrm{~s}^{-1}$ in about $30 \mathrm{~s}$ and then remains between 600 and $700 \mathrm{~km} \mathrm{~s}^{-1}$. The pressure of the evaporation front along the $Z$ axis increases by another factor 5 , reaching a value $\sim 10$ dyne $\mathrm{cm}^{-2}$ at the base of the corona. The density front reaches the central position of the heating along the $Z$ axis in $\sim 30 \mathrm{~s}$, and a height $Z \sim 10^{10} \mathrm{~cm}$ at the end of the heating $(t=150 \mathrm{~s})$, corresponding to an average propagation speed of $\sim 600 \mathrm{~km} \mathrm{~s}^{-1}$. As a consequence the density in the central post-shock region increases above a factor 3 but practically in all the coronal region it is $<10^{9} \mathrm{~cm}^{-3}$ at any time.

Given the highly localized heating release in a non confined atmosphere, the evaporation front, which mostly determines the soft X-ray burst, is not plane-parallel: it is practically semicircular, stronger along the $Z$ axis and weaker and weaker moving farther and farther from $R=0$. The reason is that the conduction front arrives at the chromosphere first along the central $Z$ axis, and progressively

\footnotetext{
1 The code does not include the effect of plasma viscosity, which has been shown to be important in solar flares and may spread out and therefore inhibit the shock front.
} 

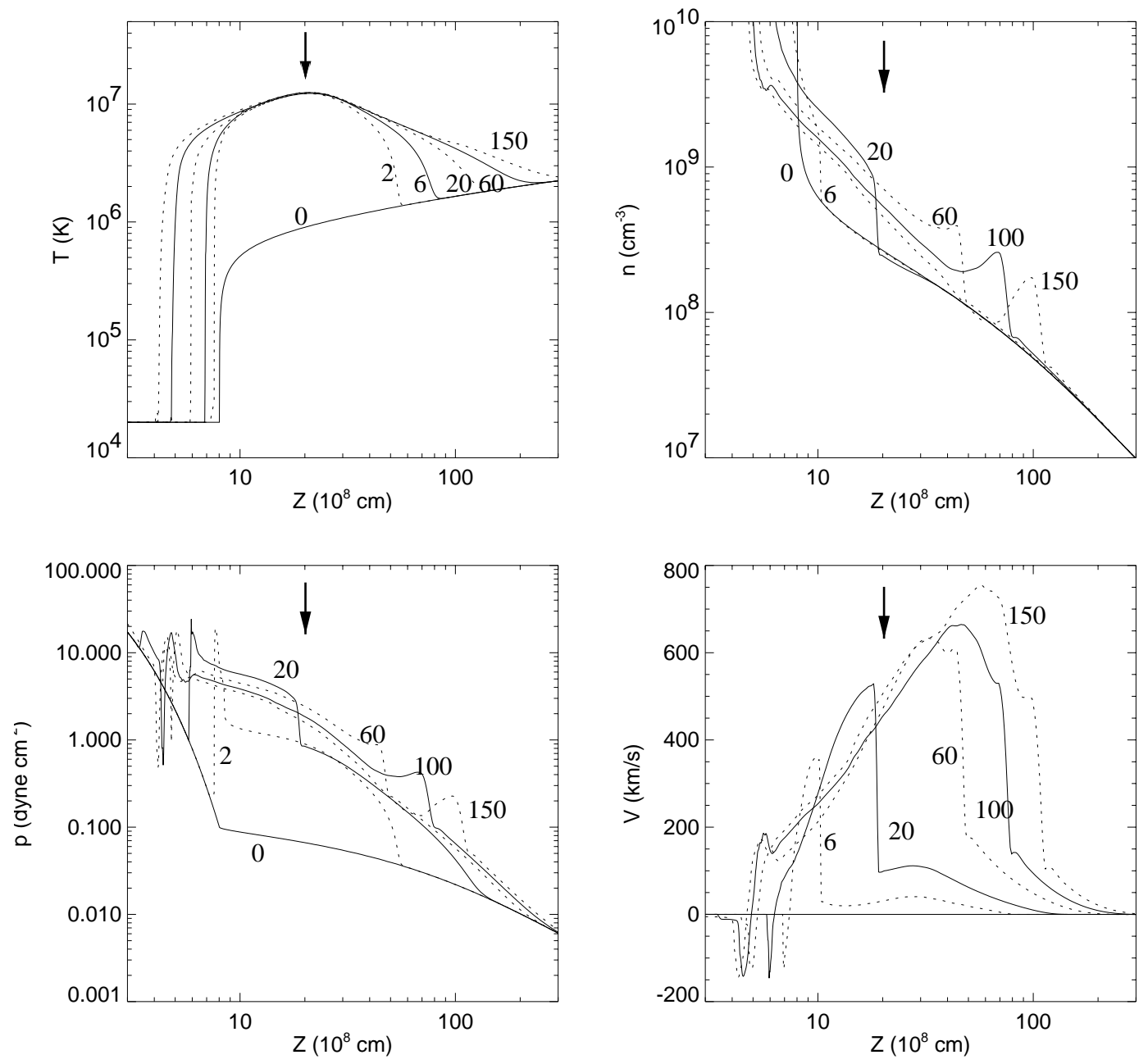

Fig. 2. Distributions of temperature $T$, density $n$, pressure $p$, and vertical component of velocity $V=V_{Z}$, along the central $Z$ axis $(R=0)$ and at various times (s) during the heating phase of the reference simulation of the non-confined flare. The arrows indicate the position of the center of the heating distribution.

later at greater and greater distances from it. The front amplitude gets weaker and weaker and with a smaller pitch angle. For $R>10^{10} \mathrm{~cm}$ the evaporation speed is $<100 \mathrm{~km} \mathrm{~s}^{-1}$ and the local density enhancement very small at any time.

The density in the post-shock region is not uniform: the plasma behind the shock moves faster than at the shock and accumulates there; the evaporation front becomes then a bow front and a region of low density (a proper depression) forms behind it. The bow front fades farther and farther from the central head. The diameter of the front is less than $2 \times 10^{10} \mathrm{~cm}$ during the whole heating phase.

\subsubsection{The decay phase}

Figure 3 shows distributions of temperature, density, pressure and vertical component of velocity along the central $Z$ axis at various times of the decay phase, in the reference simulation.
As the heating is switched off $(t=150 \mathrm{~s})$, the temperature suddenly decreases in the heating region: it halves (from 12 to $6 \mathrm{MK}$ ) in about $5 \mathrm{~s}$ at the center of the heating release. The cause is the cooling by thermal conduction, particularly efficient because the density of the hot plasma remains relatively low $\left(<10^{9} \mathrm{~cm}^{-3}\right)$. The cooling front propagates radially from the heating region, while the internal temperature gets lower and lower. The hot front produced by the heating impulse has instead weakened and practically disappeared when reaching the higher and relatively hotter (but very thin) corona. After one minute since the heating switch off $(t \approx 210 \mathrm{~s})$, the temperature along the $Z$ axis overshoots below the initial temperature of $\approx 1 \mathrm{MK}$. At $t=300 \mathrm{~s}$ only a shell at $\sim 10^{10} \mathrm{~cm}$ from the system origin, and $\sim 10^{10} \mathrm{~cm}$ thick, is still (slightly) hotter than the initial atmosphere, while the originally heated region is practically all cooler than $1 \mathrm{MK}$.

While such a fast cooling is occurring in the heated region, the evaporation front continues to propagate upwards and outwards. The shell expands but maintains more or less the same shape, becoming geometrically 

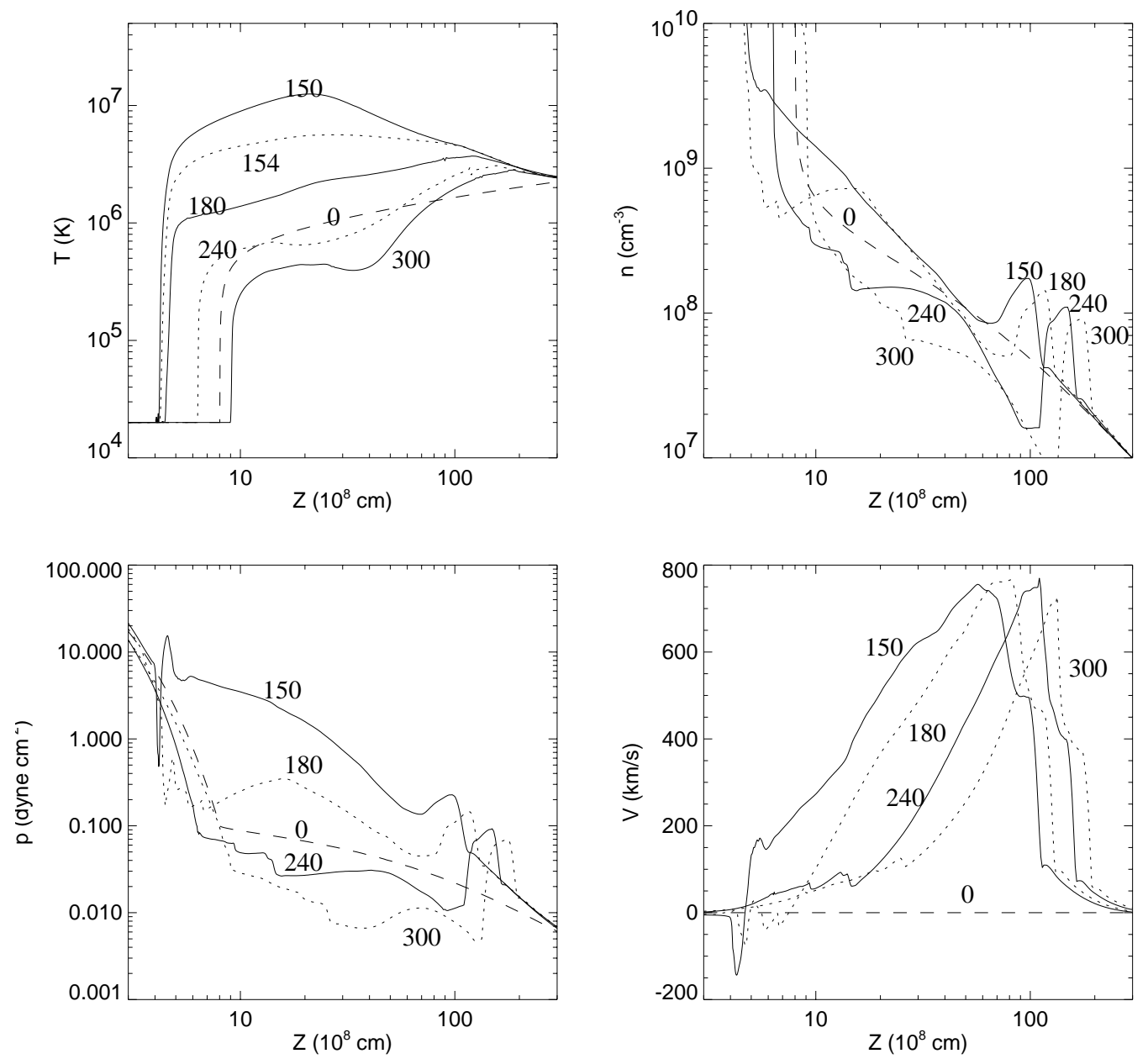

Fig. 3. As in Fig. 2 but taken at times after the heating is switched off.

thicker and thicker. The head front is at speed around $400 \mathrm{~km} \mathrm{~s}^{-1}$, the back front is steadily slightly below $800 \mathrm{~km} \mathrm{~s}^{-1}$. The difference in speed causes the accumulation.

The depression formed behind the head front deepens and expands continuously behind the evaporation front after the heating is switched off. At first it is confined to a rather small region behind the head front, but it extends for $\sim 10^{10} \mathrm{~cm}$ below the head front at $t=300 \mathrm{~s}$.

At this time, the thickness of the head front, with a density $\sim 5$ times higher than the background atmosphere, is around $5 \times 10^{9} \mathrm{~cm}$; the front has reached a height $Z \sim 2 \times 10^{10} \mathrm{~cm}$ above the stellar surface and has a diameter (distance from the $R=0$ axis) of $\sim 1.5 \times 10^{10} \mathrm{~cm}$. The density in the head front reduces from $\sim 3 \times 10^{8} \mathrm{~cm}^{-3}$ to $\sim 10^{8} \mathrm{~cm}^{-3}$ as it moves away, while the region behind gets less and less dense, the core of it decreasing below $10^{7} \mathrm{~cm}^{-3}$. The pressure in that core, but even below it, in a layer $10^{10} \mathrm{~cm}$ thick, is around 0.01 dyne $\mathrm{cm}^{-2}$, $1 / 10$ the initial one.

After $t=300 \mathrm{~s}$ the cooling of the central regions and the expansion of the evaporation shell progress with no new feature to remark. At $t=800 \mathrm{~s}$ no temperature enhancement is visible anywhere, while the evaporation front is still well evident with the top above $3 \times 10^{10} \mathrm{~cm}$ from the surface, a height where the density of the background corona is below $10^{7} \mathrm{~cm}^{-3}$, and the tail all above $10^{10} \mathrm{~cm}$. The diameter of the shell is $\sim 5 \times 10^{10} \mathrm{~cm}$, its maximum thickness $\sim 1.5 \times 10^{10} \mathrm{~cm}$, but the density is not uniform inside, ranging between $3 \times 10^{7} \mathrm{~cm}^{-3}$ (ahead) and $10^{7} \mathrm{~cm}^{-3}$ (behind). The speed of the head front is decreasing to $200 \mathrm{~km} \mathrm{~s}^{-1}$. The gap behind the front appears to be gradually filling and reducing.

\subsection{The $X$-ray emission of the reference flare}

From the model results we have synthesized the X-ray emission both in the ASCA/SIS band and in two relevant X-ray lines, namely Mg XI at $9.169 \AA$ and Fe XXI at $128.752 \AA$ with peak formation temperatures of $6.5 \mathrm{MK}$ and 10.5 MK, respectively. These two lines are representative of strong emission lines typically visible in the band of grating detectors such as those on board Chandra and XMM-Newton and with formation temperatures relevant to become significant lines during the reference flare. In the longer wavelength line the spectrometers typically have higher spectral resolution. 
2. $s$

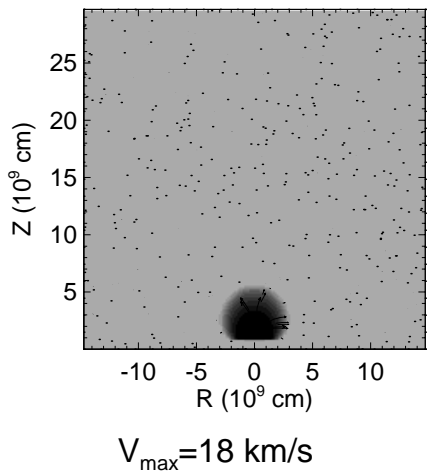

50. s

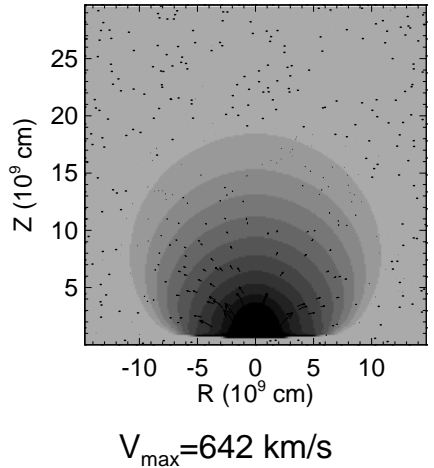

154. $\mathrm{s}$

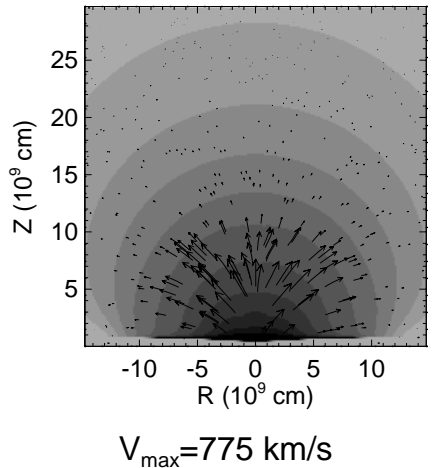

10. $\mathrm{s}$

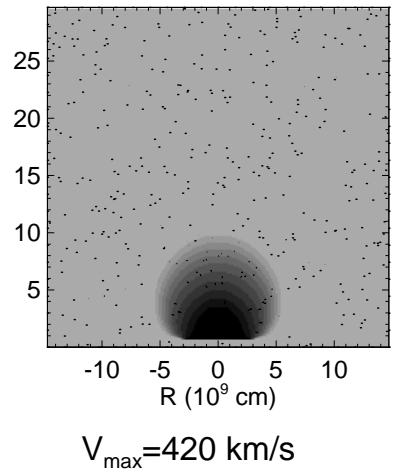

100. $\mathrm{s}$

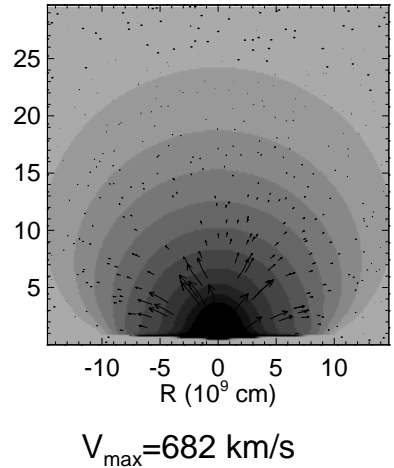

200. $s$

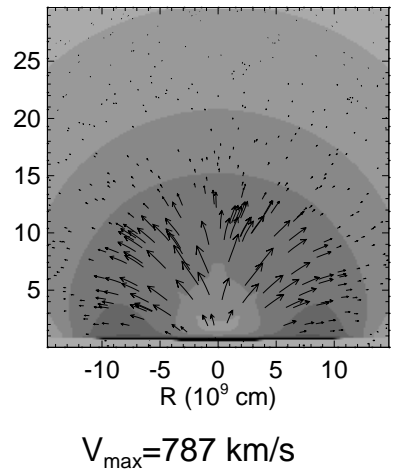

30. $s$

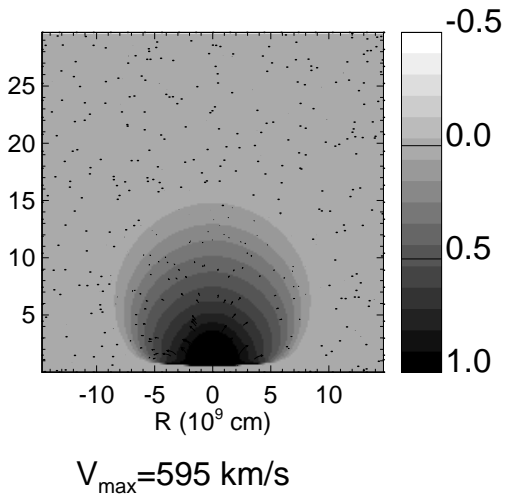

150. $\mathrm{s}$

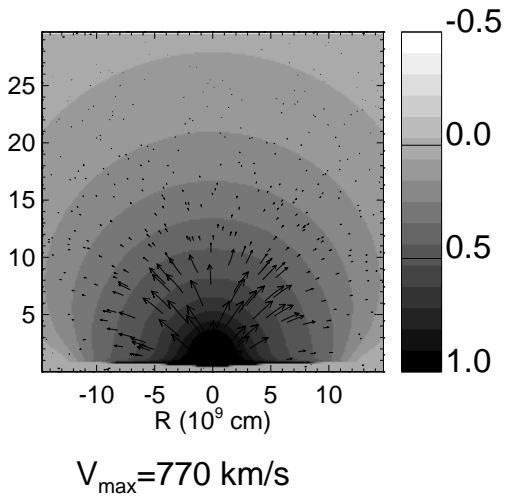

300. $\mathrm{s}$

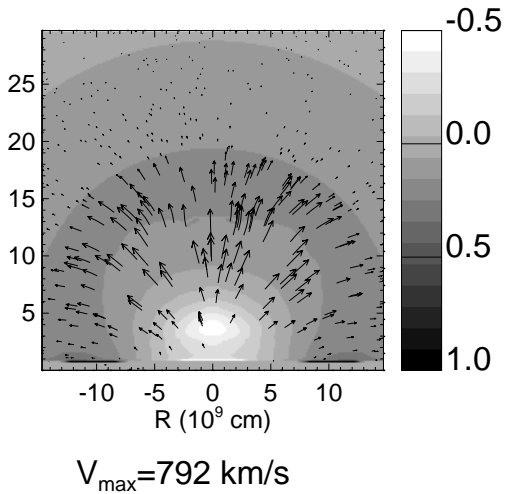

Fig. 4. Grey scale images of the temperature contrast $T / T_{0}$, where $T_{0}$ is the temperature distribution of the unperturbed atmosphere, and velocity field (arrows) at various times during the reference simulation. It is shown the region around the heating deposition. The grey-scale is logarithmic. The maximum speed corresponding to the longest arrow is shown below each image.

The X-ray spectra at the focal plane of the ASCA/SIS and in the two selected lines, have been computed in the whole computational domain and at each output time, according to:

$\mathcal{E}_{e s}(E, r, z, t)=n^{2}(r, z, t) G(E, T(r, z, t))$

where $G(E, T)$ is the emissivity per unit emission measure and per unit photon energy in the case of the ASCA/SIS, and per unit Angstrom in the case of the spectral lines. The emissivity in the ASCA/SIS band from 0.6 to $14 \mathrm{keV}$ has been obtained by folding the ASCA/SIS effective area and response with Raymond \& Smith (1977) spectra of isothermal plasma, assuming full Anders \& Grevesse metal abundances and a small interstellar medium absorption $\left(N_{\mathrm{H}}=10^{18} \mathrm{~cm}^{-2}\right)$. The emissivity in the two spectral lines have been extracted from the CHIANTI database (version 3.02, Dere et al. 1997), assuming ionization equilibrium computed according to Arnaud and Raymond (1992) and solar metal abundances (Grevesse \& Anders 1991) and using the default density of $10^{10} \mathrm{~cm}^{-3}$. 
2. $s$

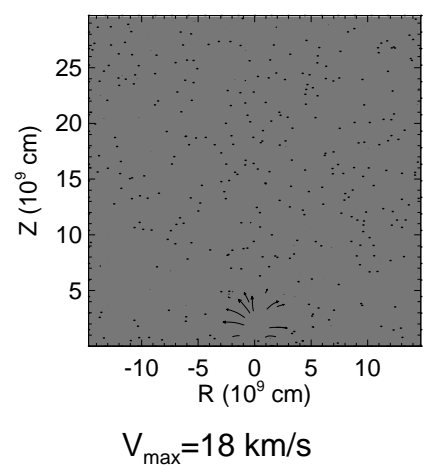

50. $\mathrm{s}$

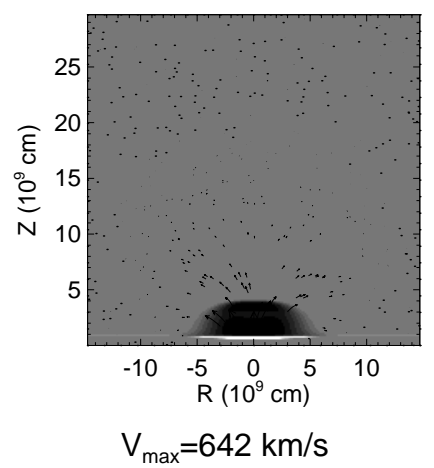

154. $\mathrm{s}$

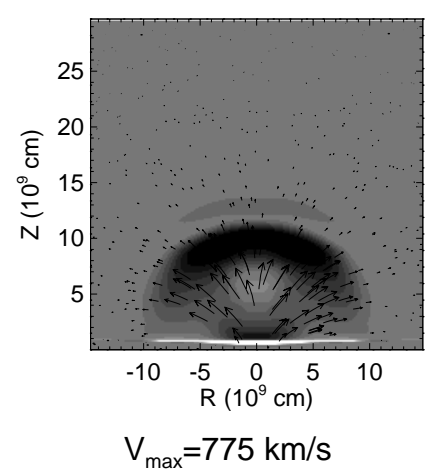

10. $\mathrm{s}$

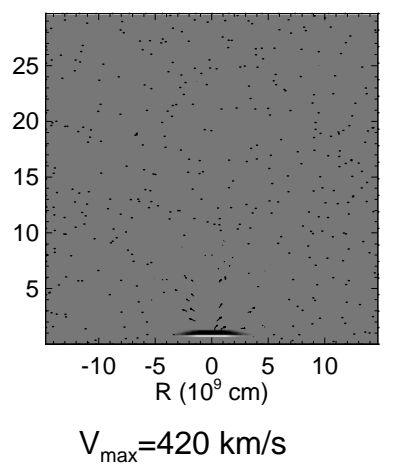

100. $\mathrm{s}$

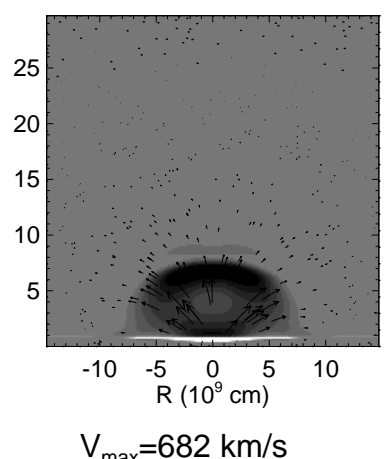

200. $\mathrm{s}$

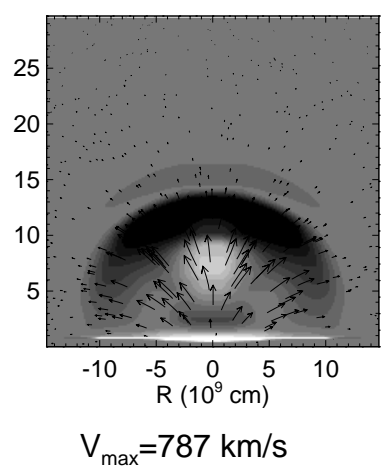

30. $s$

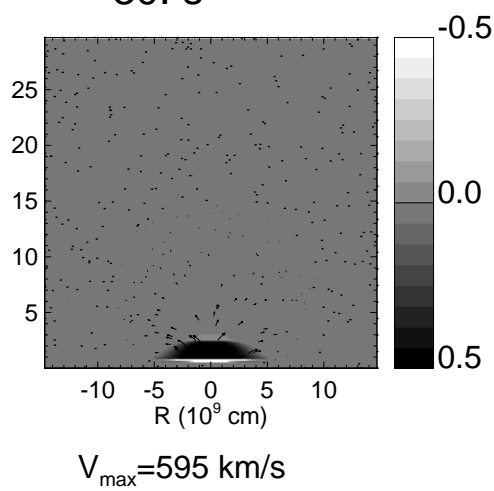

150. $\mathrm{s}$

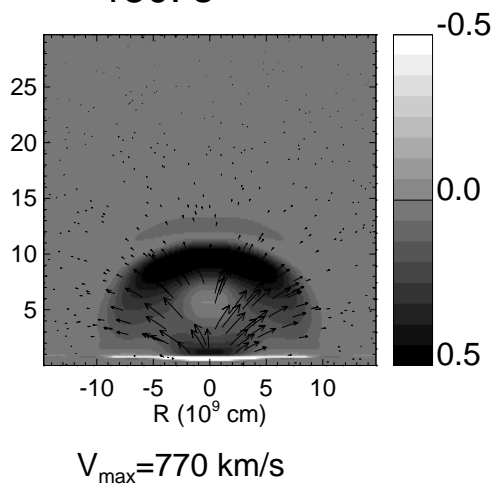

300. $\mathrm{s}$

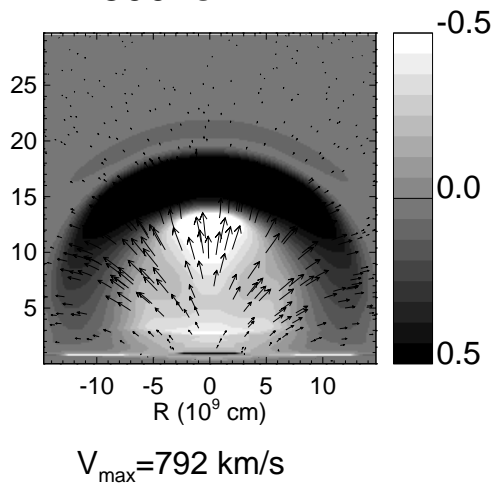

Fig. 5. As in Fig. 4 but for the density contrast $n / n_{0}$, where $n_{0}$ is the density distribution of the unperturbed atmosphere.

The spatial distribution of the total focal-plane emission per unit volume at each time is then obtained by integrating in energy:

$\mathcal{E}_{\mathrm{s}}(r, z, t)=\int_{E_{\min }}^{E_{\max }} \mathcal{E}_{\mathrm{es}}(E, r, z, t) \mathrm{d} E$.

Figure 6 shows, for the reference simulation, cross-sections of the resulting distributions of plasma emission in the ASCA/SIS band at the same times and in the same region as Figs. 4 and 5. The cross-sections are taken on a plane across the $Z$ axis. The emission at early times is clearly concentrated in the heating region and mostly in a thin layer at the base of corona. Until $t=30 \mathrm{~s}$ an almost spherical weak emission front expands progressively and is clearly associated to the propagation of the thermal front (see Fig. 4). After $t=30 \mathrm{~s}$ another brighter front rises from the base of the corona, with a less regular shape, and is due to the density front evaporating from the chromosphere (see Fig. 5). Soon after the heating is switched off $(t=154 \mathrm{~s})$, the bright knot at the base of the corona rapidly fades, due to the sudden temperature drop described in Sect. 3.2.2. At $t=200 \mathrm{~s}$ most of the emission comes from the evaporating front, which continues to expands but gradually fades away $(t=300 \mathrm{~s})$.

Since the flare would not be resolved at stellar distances, we have then integrated the emission distribution over the relevant region of the computational domain to obtain the light curve at the focal plane of ASCA/SIS and the flux at Earth in the two selected spectral lines:

$\mathcal{E}(t)=\int_{V} \mathcal{E}_{\mathrm{s}}(r, z, t) \mathrm{d} V$ 
2. $\mathrm{S}$

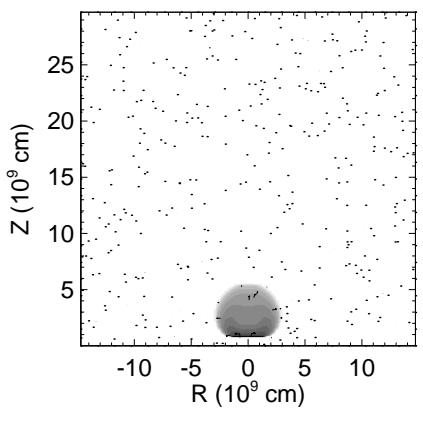

$\mathrm{V}_{\max }=68 \mathrm{~km} / \mathrm{s}$

50. $\mathrm{s}$

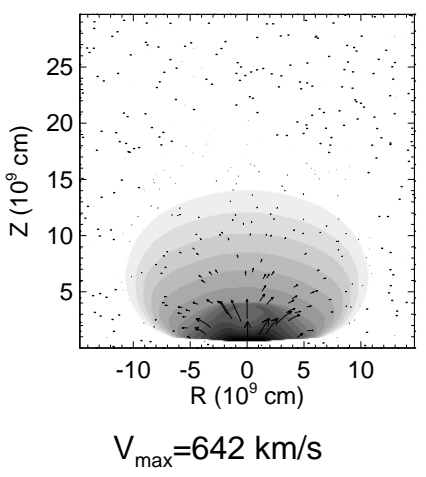

154. $s$

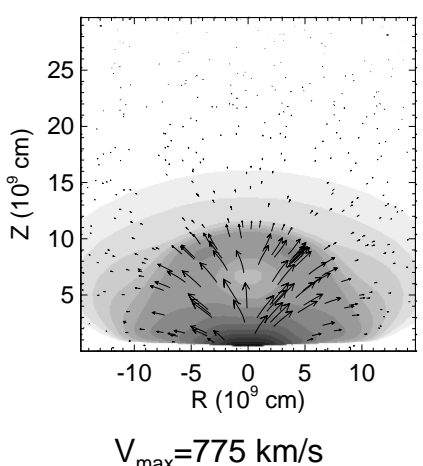

10. $\mathrm{s}$

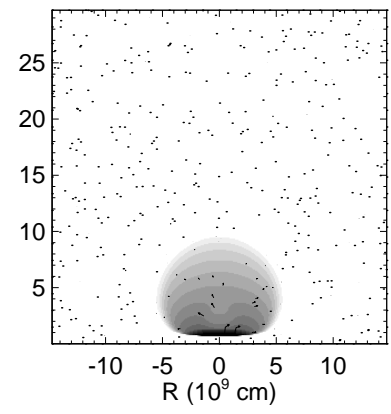

$\mathrm{V}_{\max }=420 \mathrm{~km} / \mathrm{s}$

100. $\mathrm{s}$

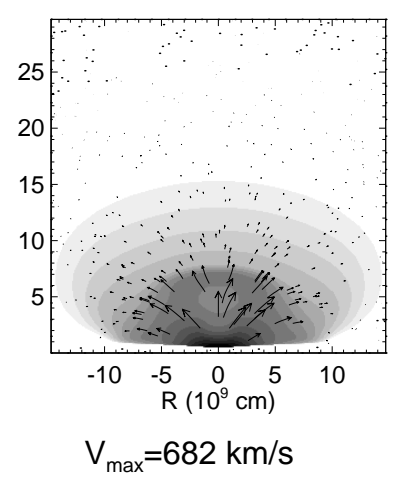

200. $\mathrm{s}$

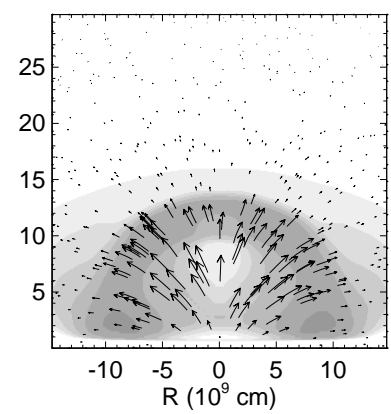

$\mathrm{V}_{\max }=788 \mathrm{~km} / \mathrm{s}$
30. $s$

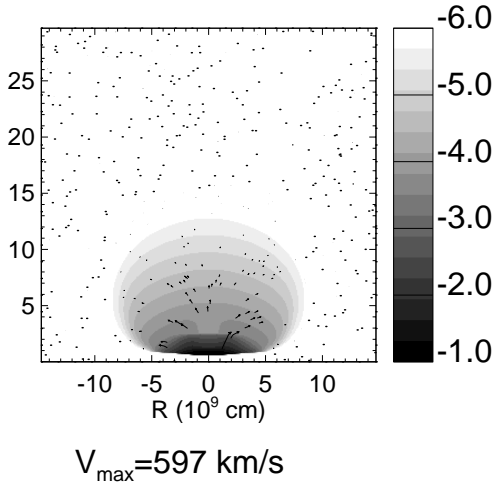

150. $\mathrm{s}$

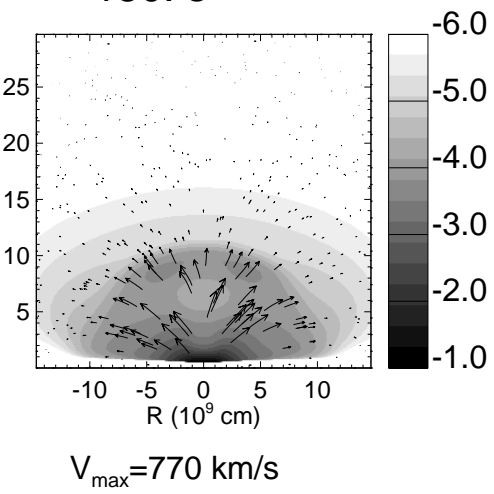

300. 5

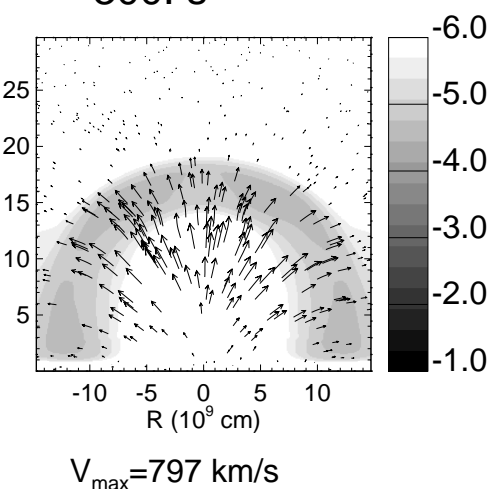

Fig. 6. Cross-sections (grey scale) of the X-ray emission (logarithmic scale normalized to global maximum) distribution in the ASCA/SIS band on a plane across the $Z$-axis and in the region where the heating is released, and velocity field (arrows) at various times during the reference simulation. Region, grey scale and maximum speed as in Fig. 4.

where $V=\pi R_{\max }^{2} Z_{\max }$ is the domain volume. In deriving the focal-plane light curve $\mathcal{E}(t)$ we have assumed a standard distance of the flaring source of 1 pc from the detector.

Figure 7 shows the flare net light curves at the ASCA/SIS focal plane, obtained by subtracting the count rate at the initial time of each simulation, taken as background non-flaring emission, for all cases reported in Table 1, including the model of confined flare.

Let's first focus the attention to the light curve of the reference case (the lowest one) and compare it with the confined one: it looks quite different. The light curve of the confined flare is characterized by a relatively rapid rise phase, a well-defined peak and a gradual decay. The light curve of the modeled unconfined flare, instead, after a very rapid rise $(\sim 20 \mathrm{~s})$ to the maximum value $(\sim 5$ times the initial emission level), shows a long plateau, staying virtually constant, with small fluctuations $(\sim 20 \%)$, until the end of the heating $(150 \mathrm{~s})$. As soon as the heating is switched off, the light curve suddenly drops: the emission halves in less than one minute. We will call this phase Fast decay. Since then on, the emission decreases more gradually with an e-folding decay time of $\sim 5 \mathrm{~min}$, as reported in Table 1. We will call this phase Gradual decay. We notice that the light curve of the non-confined flare follows much more closely the evolution of the heating: in particular, 


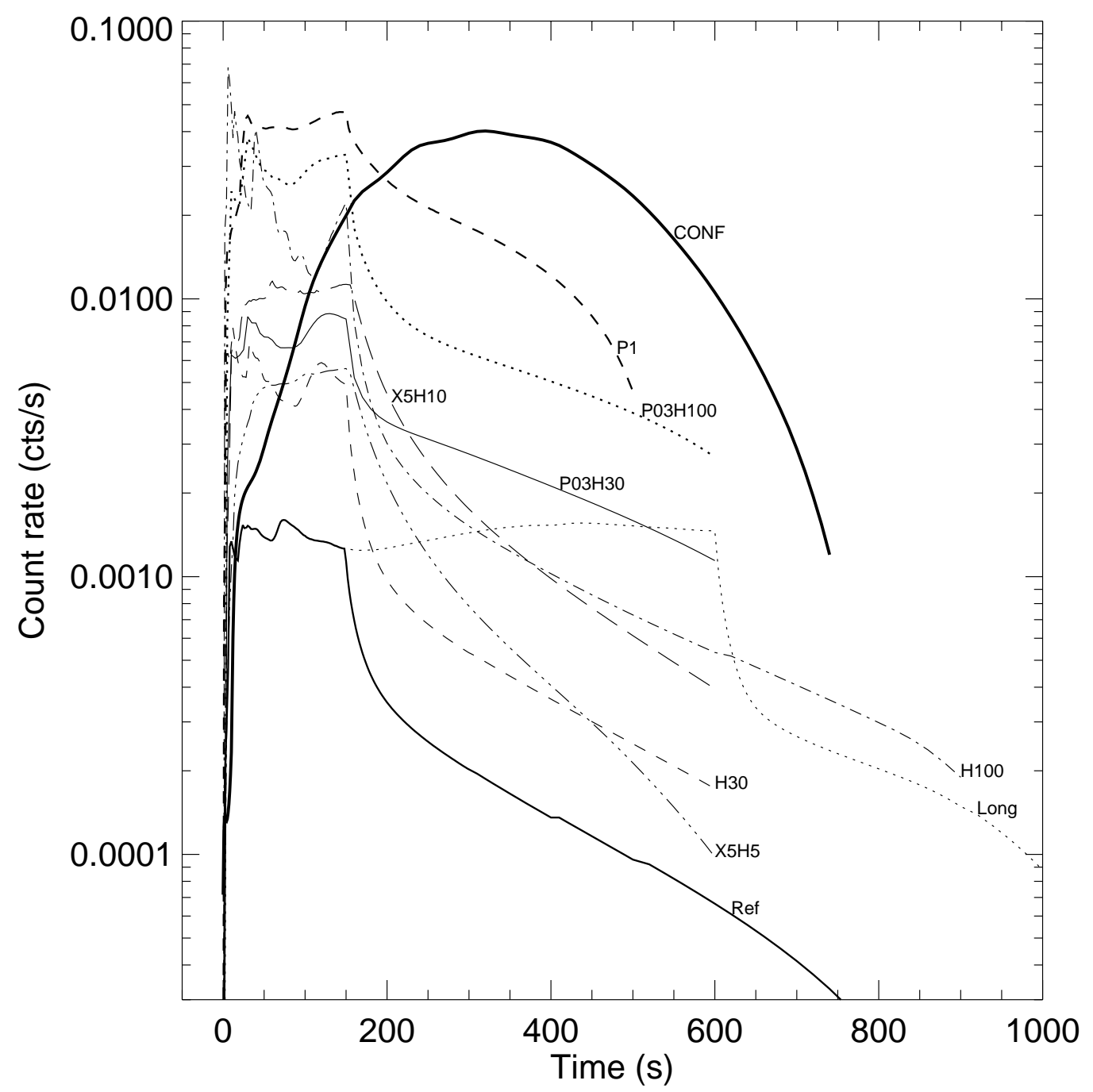

Fig. 7. Light curves at the ASCA/SIS focal plane, assuming a distance of 1 pc, synthesized from the results of the simulations listed in Table 1. The light curve of a modelled confined flare is also shown for comparison (thickest solid line, labelled CONF).

the emission drops as soon as the heating stops whereas, in the confined case, it keeps on increasing and begins to decay only $\sim 200$ s after the end of the heating. The decay trends are complementary: in the confined case it is slow at first and then gradually becomes faster and faster, also because more and more plasma cools and is no longer visible in the instrument passband.

We have made the exercise of a $\chi^{2}$ fit of ASCA/SIS spectra sampled along the light curve with isothermal plasma spectra, a standard analysis in stellar coronal physics. We obtain a sequence of temperature and normalization (emission measure) values which can be put in a density-temperature diagram (where the square root of the emission measure is used as proxy of the density, as it has been typically done for confined flares ${ }^{2}$, e.g. Sylwester et al. 1993; Reale et al. 1997), in order to compare this work with previous work to model stellar flare decay (Reale et al. 1997; Reale \& Micela 1998; Favata \& Schmitt 1999). This diagram is used as a diagnostics of

\footnotetext{
${ }^{2}$ Notice that for non-confined flaring plasma, this assumption, based on constant volume, is grossly incorrect.
}

the possible presence of sustained heating during the decay (Sylwester et al. 1993).

The upper panel of Fig. 8 shows the path of the reference non-confined flare in the density-temperature diagram, and, for comparison, the one of the confined flare. We first notice that the maximum best fit temperature of the non-confined flare $(\sim 4 \mathrm{MK})$ is much lower than the true maximum temperature (see Table 1 ), by more than a factor 2. The reason is that the best-fit temperature is a weighted average over the emitting region, which is much larger and cooler than the directly heated region; in the latter the temperature is close to the plasma maximum temperature. We end up with an "observed" temperature well below typical flare temperatures, although there is plasma above $10 \mathrm{MK}$ and the rate of energy released is enough to produce a medium (M GOES class) solar flare, as the confined one shown in the figure, with peak plasma temperatures around $20 \mathrm{MK}$. The emission measure values of the reference model are lower, by a factor 5 , than those of the confined model flare, with a maximum value of $\sim 3 \times 10^{47} \mathrm{~cm}^{-3}$ versus $\sim 1.6 \times 10^{48} \mathrm{~cm}^{-3}$. 

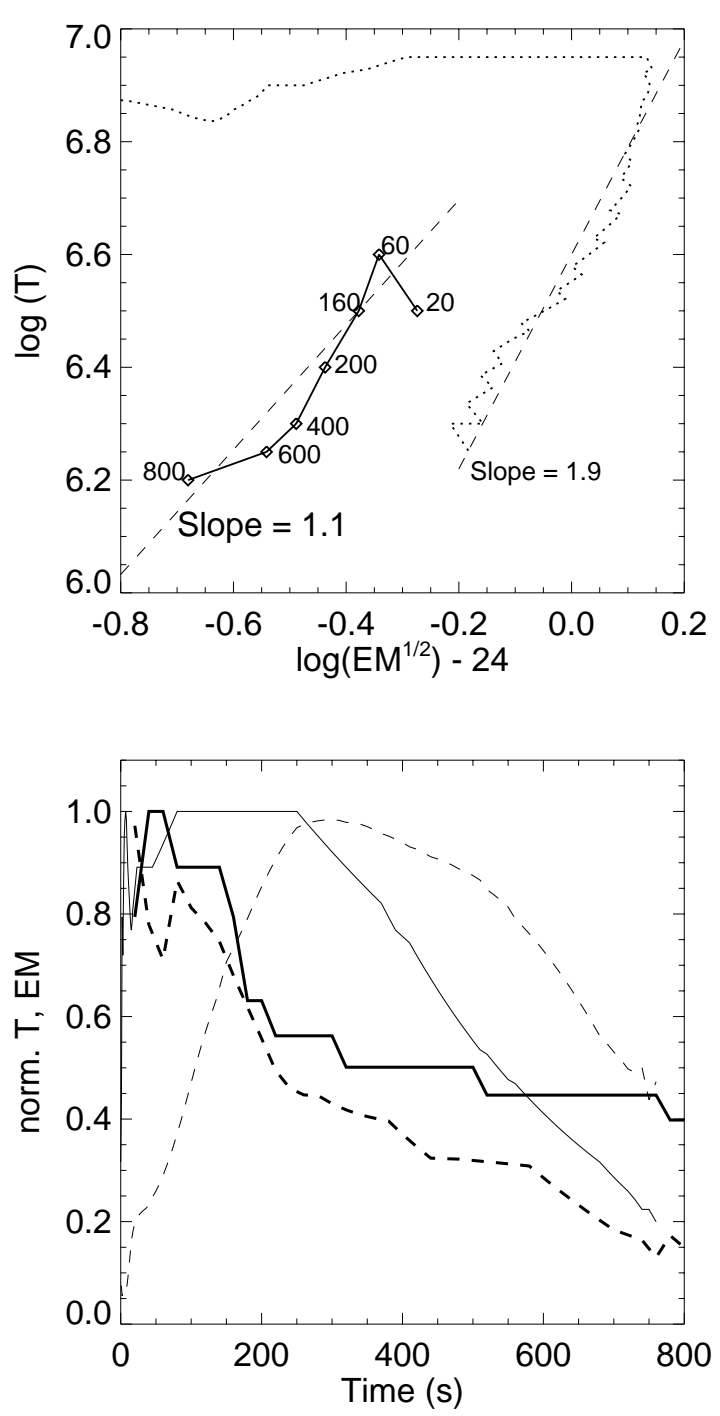

Fig. 8. Upper panel: path in the density-temperature diagram obtained by fitting the ASCA/SIS spectra synthesized from the reference model results with isothermal plasma models at various times (s) during the computed evolution (solid line). The dashed line has the labeled slope and well describes the path of the flare decay. The path of the confined flare model is also shown for comparison (dotted line). Lower panel: evolution of the temperature (solid lines) and of the emission measure (dashed lines) of the reference model (thick lines) and of the model of confined flare (thin lines). For the sake of clarity, all quantities are normalized to 1: the normalization factors are 4.0 MK and 8.9 MK for temperature, respectively, and $0.3 \times 10^{48} \mathrm{~cm}^{-3}$ and $1.4 \times 10^{48} \mathrm{~cm}^{-3}$ for emission measure.

The flare path in the diagram is different from the one of the confined flare in the heating phase: the emission measure reaches very soon its maximum and then gradually decreases (leftwards), and the temperature slightly fluctuates, whereas the model of confined flare shows increasing emission measure at constant temperature (Jakimiec et al. 1992), as also observed in many solar flares (Sylwester et al. 1993). The path is much more "standard" in the decay phase: temperature and emission measure both decrease, along the line with positive slope,

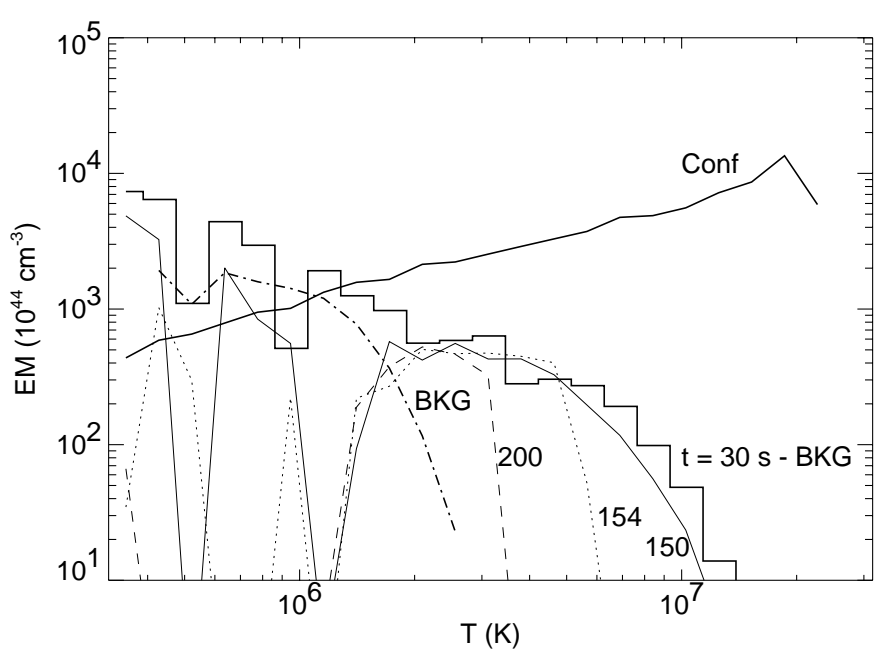

Fig. 9. Distributions of emission measure vs temperature of the reference model at various times during the flare evolution: in the heating phase $30 \mathrm{~s}$ (histogram), $150 \mathrm{~s} \mathrm{(solid} \mathrm{line);}$ in the decay phase $154 \mathrm{~s}$ (dotted line), $200 \mathrm{~s}$ (dashed line). The distributions shown are subtracted by the initial unperturbed distribution at time $(t=0 \mathrm{~s})$, also shown for comparison (dashed-dotted thick line). A distribution obtained from the model of confined flare (at the end of the heating phase, $t=150 \mathrm{~s}$ ) is also shown for comparison (thick solid line).

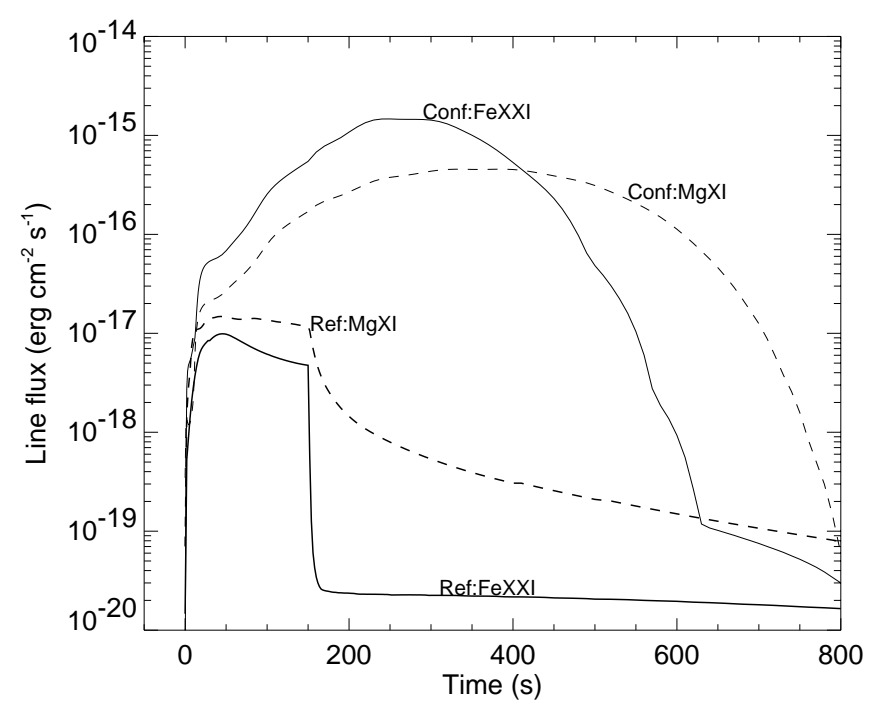

Fig. 10. Light curves in the two selected X-ray lines for the reference model and for the confined flare model.

very similar to many observed cases. The value of the slope of the non-confined case would suggest a moderately heated decay (e.g. Reale et al. 1997; Favata et al. 2000) if interpreted as a confined flare. At the end of the decay the average temperature stays relatively long at $\sim 2 \mathrm{MK}$.

The unusual trend of the $n-T$ path in the heating phase is directly connected to the relative timing of the evolution of the density and the temperature (shown in the lower panel of Fig. 8). In confined flares, the temperature reaches its maximum and begins to decay well earlier than the emission measure; the time delay is $\sim 200 \mathrm{~s}$ in the case shown. This does not occur in the reference model 
of non-confined flare (as well as in all the other models in Table 1): the emission measure is at its maximum very soon, simultaneously to the temperature, and its evolution is similar to the temperature evolution and on similar time scales.

In addition to the different timing of the temperature and emission measure evolution, also the distributions of the emission measure versus temperature during the evolution of the non-confined model flare shows remarkable differences from corresponding ones during the evolution of the confined model flare. Figure 9 shows some distributions of the emission measure versus temperature $(E M(T))$ sampled at various times during the reference model of non-confined flare as compared to one $E M(T)$ obtained from the confined flare model at the end of the heating phase $(t=150 \mathrm{~s})$. In order to evaluate the effect of the flare heating on the plasma, the figure shows the $E M(T)$ profiles of the reference model subtracted of the unperturbed pre-flare $E M(T)$ profile. In agreement to Fig. 8, the maximum amount of emission measure is present during the heating phase (see profiles at $t=30 \mathrm{~s}$ and $t=150 \mathrm{~s}$ ). The effect of the heating is to add emission measure at temperatures above $2 \mathrm{MK}$, up to $\sim 10 \mathrm{MK}$. Interestingly, the heating produces additional, and even larger, components of emission measure at lower temperatures. The overall $E M(T)$ profile in the heating phase of the reference model therefore decreases with temperature, according, approximately, to a power law of index $\sim-1.5$. This trend is qualitatively different from the typical $E M(T)$ profiles of flaring (and non-flaring) loops, which instead typically increase with temperature with an index $\sim 1.5$. As the heating is switched off, the hottest part of the $E M(T)$ suddenly disappears $(t=154 \mathrm{~s})$, and at $200 \mathrm{~s}$ there are no pre-flare components hotter than 4 MK. Furthermore, gaps of emission measure form for $T \leq 1 \mathrm{MK}$ : the plasma in the heated region cools and only the plasma in the outward expanding shell contributes to the emission measure in excess of the background.

As shown in Fig. 9, emission measure components are present in a wide range of temperatures during the heating phase and, therefore, we can expect significant emission in the selected spectral lines. Figure 10 shows the light curves of the reference non-confined flare in the selected $\mathrm{Mg}$ XI at $9.169 \AA$ and Fe XXI at 128.752 $\AA$ lines, assumed always in equilibrium of ionization. The corresponding light curves for the confined model flare are also shown for comparison. The light curves of the reference flare globally resemble the other synthesized ones at the ASCA/SIS focal plane (Fig. 7). Their decay starts simultaneously. While the light curve in the hotter Fe XXI line drops by more than two orders of magnitude in less than $5 \mathrm{~s}$, due to the sudden cooling of the heated region, the Mg XI line decays more slowly, because emission measure survives longer at temperatures below $10 \mathrm{MK}$. Once again, the light curves look quite different from the corresponding ones of the confined flare model. In particular, the cooler Mg XI line peaks later than the hotter Fe XXI line, which is more directly sensitive to the evolution of the heating. The shapes

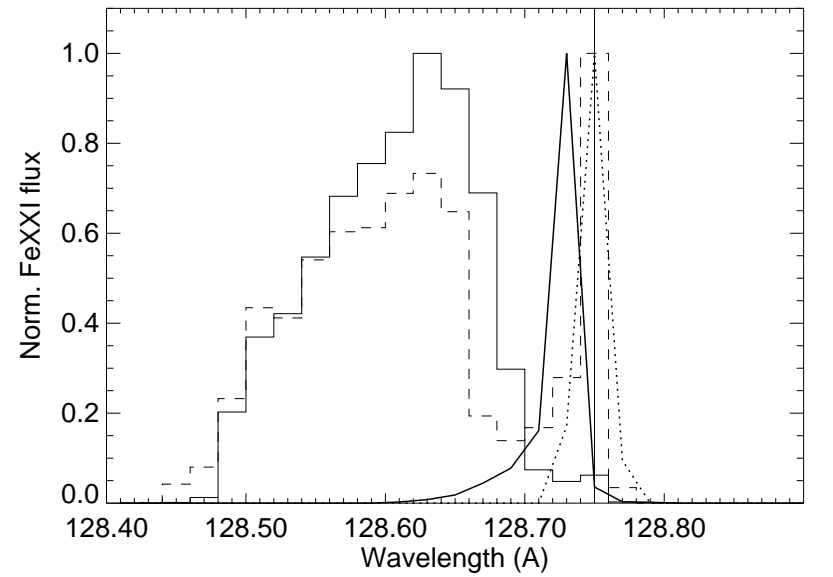

Fig. 11. Fe XXI $128.75 \AA$ line profiles synthesized from the reference model and averaged over the heating phase (from 0 to $150 \mathrm{~s}$, solid histogram), and over the next $150 \mathrm{~s}$ in the decay phase (dashed histogram). Analogous profiles from the confined model, averaged over the heating phase (from 0 to $150 \mathrm{~s}$, solid line) and over the next $150 \mathrm{~s}$ (dashed line) are also shown for comparison. The normalization factors are $3.9 \times 10^{-19}, 6.0 \times$ $10^{-22}, 8.0 \times 10^{-17}, 2.9 \times 10^{-16} \mathrm{erg} \mathrm{cm}^{-2} \mathrm{~s}^{-1} \AA^{-1}$, respectively. The spectral bin is $0.02 \AA$. The nominal line wavelength is marked (vertical line).

of both the line decays for the reference flare are different from those of the confined flare, but it may not be trivial to discriminate between them in real data, typically coarsely resolved in time, and sometimes with small signal to noise ratio.

As discussed in Sect. 3.2, the evolution of the nonconfined plasma has showed the presence of a steady upward moving plasma front, with speed of several hundreds $\mathrm{kms}^{-1}$. If favorably oriented with respect to the line of sight, we may expect such high speed plasma component to produce significant Doppler blue-shifted components in resolved lines. Figure 11 shows two detailed profiles of the Fe XXI $128.75 \AA$ line synthesized from the reference model, in the assumption that the flare is "diskcentered" with respect to the observer. One profile is averaged over the heating phase (from 0 to $150 \mathrm{~s}$ ), and the other over the next $150 \mathrm{~s}$ in the decay phase. The former profile is practically entirely blue-shifted, while the other also shows a significant static component. The blue-shifted components both peak at $128.63 \AA$, corresponding to an outward speed component of $\sim 300 \mathrm{~km} \mathrm{~s}^{-1}$, and are considerably broadened, with a width of $0.05-0.10 \AA$ (100$200 \mathrm{~km} \mathrm{~s}^{-1}$ ). Such persistent blue-shifted components are not present in the profiles from the model of confined flare. The profile averaged over the whole heating phase is slightly blue-shifted $\left(0.02 \AA, 50 \mathrm{~km} \mathrm{~s}^{-1}\right)$, due to the plasma evaporation, the other profile is practically unshifted. Both profiles are much sharper than those of the reference model. Indeed considerable blue-shifted components (at speed $\geq 100 \mathrm{~km} \mathrm{~s}^{-1}$ ) are predicted also by models of confined flares, but only in the very initial phase of the plasma evaporation, generally within the first $60 \mathrm{~s}$ in an active region loop. Then the blue-shift is washed out by 
the next more gradual, less dynamic (but much brighter) evaporation.

\subsection{The other simulations}

As discussed in Sect. 3.1, the basic free parameters of the simulations are the pressure at the base of the corona and the heating parameters. The explored values are shown in Table 1 . We have already described in detail the results of the reference simulation. The plasma evolution in all the other simulations invariably resembles the one obtained in the reference case, i.e. initial temperature increase and expanding conduction front, subsequent bow density front expanding upwards from the chromosphere, decay with very fast temperature decrease and continuous expansion of the density bow front. However several quantitative differences occur. First of all, the maximum temperature is sensitive to the heating parameters and, in particular, it increases with the rate per unit volume $H_{0}$ and with the height $Z_{\mathrm{H}}$. Given the high efficiency of thermal conduction, the temperature in a flare reaches an equilibrium value very rapidly. In steady-state closed coronal structures (loops) the plasma maximum temperature is linked to the heating amount by coronal scaling laws (Rosner et al. 1978, hereafter RTV). One may then use the temperature of a loop to estimate the heating rate with the aid of the scaling laws (e.g. Reale et al. 2000; Maggio et al. 2000). In the presence of the same physical effects at work, we have applied the scaling laws also to our configuration despite the scaling laws were devised for closed one-dimensional systems.

By combining the maximum temperature scaling law to the volume heating scaling law we obtain:

$L \approx 10^{-3} T^{7 / 4} H^{-1 / 2}$

where $L$ was the loop half length in the RTV scaling laws, $T$ is the plasma maximum temperature and $H$ is heating volume rate. If we assume $T=T_{\max }$ and $H=H_{0}$, we invariably obtain $L \sim 10^{9} \mathrm{~cm}$ for the cases named Ref, H30 and H100 in Table 1 and $L \sim 2 \times 10^{9} \mathrm{~cm}$ for both the cases X5H5 and X5H10. From Table 1, we realize that these lengths correspond essentially to the "diameter" of the heating distributions, i.e. twice the width $\sigma_{\mathrm{H}}$. This means that in this non-confined configuration the thermal distribution of the flaring region is virtually dominated by the heating distribution.

The maximum temperatures of the simulations range between 13 and 25 MK. Notice that, in order to produce a 25 MK flare, not indeed a major one on the standard of stellar flares, we have needed quite a high heating rate of $2 \times 10^{29} \mathrm{erg} / \mathrm{s}$. We notice also a weak inverse dependence of the maximum temperature on the coronal initial base pressure: the heating is less effective in an atmosphere initially at higher pressure.

Figure 7 includes light curves at the ASCA/SIS focal plane for all simulations in Table 1, all computed as described in Sect. 3.3. Looking at the figure, the most striking feature is certainly that, apart from a scale factor clearly depending on the amount of energy released in the atmosphere, all light curves share a common shape: a very fast rise, a relatively constant phase, lasting until the heating is switched off, a sudden fast decay by a factor 2 to 5 on time scale of few seconds, followed by a more gradual decay on time scales of few hundreds of seconds. This evolution and its timing trace closely the evolution of the heating.

It is straightforward to attribute the fast initial cooling to thermal conduction. From Eq. (3), the conductive cooling time can be approximated as:

$\tau_{c} \sim 5 \frac{n_{9} l_{9}^{2}}{T_{7}^{5 / 2}} \quad \mathrm{~s}$

where $n_{9}$ is the density in units of $10^{9} \mathrm{~cm}^{-3}, l_{9}$ is a characteristic length $\left(10^{9} \mathrm{~cm}\right)$ and $T_{7}$ is the peak temperature $\left(10^{7} \mathrm{~K}\right)$. In practice, the relatively small density value makes the cooling by conduction particularly efficient. As shown by Table 1 , the low density is common to all simulations and is an intrinsic, important and characterizing feature of simulating non-confined flaring plasma, as further discussed below. Notice also that the highest pressure case (P1) shows a relatively higher density and, in fact, a relatively more gradual initial decay.

Later on, as temperature decreases, cooling by conduction becomes less and less efficient and other cooling mechanisms come into play, determining the flattening of the light curves in the gradual decay phase. In particular, in the analysis of the simulation results (Fig. 6) we have noticed that in the gradual decay phase the flare emission originates mostly from the expanding density shell. The front propagates in a medium reasonably approximable as isothermal, and, therefore, the gradual decay must be attributed to the progressive reduction of the emission measure. Since, as we have checked, the amount of plasma involved in the density front is approximately (within $\sim 20 \%$ ) unchanged with time as the expansion progresses, the emission measure in this phase is roughly proportional to density. As the front propagates upwards, it maintains approximately the same density contrast, as it occurs in strong shocks. Therefore the density of the front decreases simply because it moves upper and upper through less and less dense layers of the stratified corona. The density decreases exponentially:

$n \approx n_{0} \exp \left(z / s_{\mathrm{p}}\right) \approx n_{0} \exp \left(-t / \tau_{\mathrm{f}}\right)$

where $s_{\mathrm{p}} \approx 2 k T_{\mathrm{f}} /(m g) \sim 5000 T_{\mathrm{f}}$ is the pressure scale height of the front at temperature $T_{\mathrm{f}}$ for solar gravity, and

$\tau_{\mathrm{f}}=s_{\mathrm{p}} / V_{\mathrm{f}} \approx 300 \frac{T_{6.5}}{V_{500}}$

is an e-folding time obtained using the fact that the front moves at approximately constant speed $\left(V_{\mathrm{f}} \sim\right.$ $500 \mathrm{~km} \mathrm{~s}^{-1}$ ). The time $\tau_{\mathrm{f}} \sim 300 \mathrm{~s}$, obtained for $T_{\mathrm{f}} \sim 3 \mathrm{MK}$ 
is indeed of the order of the decay times reported in last column of Table 1.

Of course, the decaying trend changes again as soon as any of the above approximations no longer holds, e.g. as soon as the shock weakens (as in case P1 for $t \gtrsim 400 \mathrm{~s}$ ) or is very distant from the origin (as in the case named Long for $t \gtrsim 900 \mathrm{~s}$ ).

All light curves share the same fundamental differences from the light curve of the confined model flare: in particular the decay starts much earlier and starts fast and then is more gradual, whereas in the confined flare it starts slow and then steepens. It should be noted that, while the only way to have a significantly slower flare decay in the nonconfined atmosphere is to have a heating released over a much larger area (Eq. (11)), in contrast with flare heating theory (e.g. Golub \& Pasachoff 1997), the decay of confined flare can be made slower simply considering a longer loop, according to the scaling law (Serio et al. 1991):

$\tau_{\mathrm{conf}} \approx 120 \frac{L_{9}}{\sqrt{T_{7}}}$.

\section{Discussion}

Modeling a non-confined coronal flare has produced interesting results from the points of view both of pure modeling and of diagnostics, especially when compared to confined events.

We have considered a reference case of a flare triggered in an open structure by a heating pulse which would have produced a $20 \mathrm{MK}$ flare in a confined structure, namely a $40000 \mathrm{~km}$ long solar loop. Then we have explored several other cases, by increasing the heating rate, therefore approching more typical stellar flare conditions, by shifting upwards the heating location, as it may occur in spatially large stellar coronae, and by considering initial atmosphere at higher pressure, which may be present in active stars.

Table 2 shows the main results of the present study, by comparing properties of non-confined flare models with those of confined flare models and indicating the potential way of discriminating them through the analysis of observations.

The hydrodynamic evolution obtained from modeling non-confined flares is complex and involves many different aspects. The model flare is triggered by an impulsive heating in localized region of a stratified corona. This heating produces an immediate but localized temperature increase: proper hot flare conditions occur only within a relatively small region around the heating deposition (Item 1 in Table 2). This is at variance from a flare occurring in confined plasmas, which are typically entirely involved in the flare. In our case, thermal conduction has a more limited action range: the conduction front rapidly weakens due to its expansion and is not able to heat significantly plasma very far from the heating deposition place.

In spite of the dynamic flare evolution, the plasma reaches soon a steady state thermal condition, so that the maximum temperature and heating rate per unit volume are approximately ruled by a scaling law similar to RTV scaling laws for coronal loops, in which the heating scale length takes place of the loop half-length (Eq. (10), Item 2 in Table 2).

A crucial difference from confined flare events is the evolution of the chromospheric evaporation (Item 3 in Table 2). In a closed structure, plasma evaporates first rapidly with a strong density front, and then more gradually but continuously until the loop is completely filled up and is close to equilibrium conditions. This long-lasting evaporation in a confined structure allows density to reach high values $\left(\gtrsim 10^{11} \mathrm{~cm}^{-3}\right)$. The high density (together with the flat temperature distribution along the loop) makes cooling by conduction relatively inefficient, leading to decay times of hours in long loops. What happens in the non-confined cases is radically different. There is a single evaporation front, which propagates as a strong shock continuously outwards from the chromosphere below the heating place, but since plasma is not confined, it does not accumulate in spite of further evaporation from the chromosphere. In other words, even though the density in the flaring region soon becomes several times higher than it was in the unperturbed condition, it does not grow above the strong shock limit (e.g. Rosenau \& Frankenthal 1976), and it never becomes as high as in typical confined flares. This is an unavoidable property of a non-confined configuration: plasma cannot pile up and therefore cannot become very dense. This has several implications, but probably the most important one, especially from the diagnostical point of view, is that, as soon as the heating stops, the plasma in the heated region cools very rapidly, because thermal conduction is very efficient in a relatively low density medium. We have provided a characteristic time scale for this early and fast decay phase (Eq. (11)), which, for typical conditions, is of the order of few seconds (Item 4 in Table 2).

We have seen that, after the initial drop, the decay progresses more gradually, driven by the decrease of density of the emitting plasma contained within the expanding evaporation front, with a time scale given by the time taken by the front to move across the pressure scale height (Eq. (13)). However, even the characteristic decay time of this phase, invariably not longer than few minutes, is quite small as compared to the decay times of typical observed large stellar flares (hours or even days), ruled by the conduction/radiation cooling time (Serio et al. 1991).

The thermal and hydrodynamic evolution of the flaring plasma leads to a series of diagnostical implications, which can be searched for in observations of stellar flares. In the first place, we note that non-confined flares require much more energy to enhance the plasma temperature (Item 5 in Table 2): with the same heating rate both the maximum (see Table 1) and the average (see Fig. 8) temperatures of the non-confined flare are nearly halved with respect to the corresponding ones of the confined model. As obvious consequence one may expect long-lasting stellar flares to be also relatively cool, which is opposite to 
Table 2. Relevant properties of flare $\operatorname{models}^{a}$.

\begin{tabular}{|c|c|c|c|c|}
\hline Item & Property & Confined & Non-confined & $\begin{array}{l}\text { Diagnostic power: } \\
\text { tools }^{b}\end{array}$ \\
\hline \multicolumn{5}{|c|}{ Hydrodynamics } \\
\hline 1 & Flaring region & All loop & Localized: heated region & - \\
\hline 2 & $\begin{array}{l}\text { Scale length in RTV scal. } \\
\text { laws }\end{array}$ & Loop half-length & Diameter of heated region & - \\
\hline 3 & Evaporation evolution & Plasma accumulation & Expanding fronts & - \\
\hline \multirow[t]{2}{*}{4} & Decay time scale & $120 L_{9} / \sqrt{T_{7}}$ & early: $5 n_{9} l_{9}^{2} / T_{7}^{7 / 2}$ & - \\
\hline & & & late: $300 \sqrt{T_{6.5}} / V_{500}$ & \\
\hline \multicolumn{5}{|c|}{ Hydrodynamics \& Diagnostics } \\
\hline 5 & Flare temperature & $\operatorname{High}^{c}$ & Lower & Low: $\operatorname{LRS}^{d}$ \\
\hline 6 & Plasma density & $\operatorname{High}\left(\gtrsim 10^{11} \mathrm{~cm}^{-3}\right)$ & Low $\left(\lesssim 10^{10} \mathrm{~cm}^{-3}\right)$ & Low: $\mathrm{HRS}^{e}$ \\
\hline 7 & $T-E M$ relative timing & Peak delay & Synchronous & High: LRS \\
\hline 8 & Decay & Gradual & Fast+Gradual & Very high: $\mathrm{WLC}^{f}$ \\
\hline 9 & Line evolution & Asynchronous & Synchronous & ?: HRS \\
\hline 10 & Blue-shift phase & Short & Persistent & $\begin{array}{l}\text { Low: Very HRS }+ \\
\text { orientation }^{g}\end{array}$ \\
\hline 11 & $E M(T)$ & $\propto T^{3 / 2}$ & $\propto T^{-3 / 2}$ & ?: $\mathrm{MRS}^{h}$ \\
\hline 12 & Heating tracer & Hard X-ray l.c. & Soft X-ray l.c. & ?: WLC \\
\hline
\end{tabular}

${ }^{a}$ This table summarizes the main differences between the results of confined and non-confined flare models, discussed in more detail in Sect. 4.

${ }^{b}$ Level of diagnostic power to discriminate confined and non-confined flare: Low, High, Very high. "?" means uncertain.

${ }^{c}$ With the same heating rate: the value depends on the heating rate.

${ }^{d}$ LRS: Low Resolution Spectra required.

${ }^{e}$ HRS: High Resolution Spectra required.

${ }^{f}$ WLC: Wide-band Light Curve required.

${ }^{g}$ The flaring region must be located well inside the stellar disk.

${ }^{h}$ MRS: Medium Resolution Spectra required.

what is typically observed, with temperature even reaching $10^{8} \mathrm{~K}$ (e.g. Maggio et al. 2000). However, since one cannot exclude very high energy rates, the high temperatures observed can hardly be taken as a distinctive signature of confinement, in the light of our findings.

As discussed before, the plasma density should be instead invariably lower in non-confined flares (Item 6 in Table 2). This may be directly diagnosed with densitysensitive lines detected with high resolution spectrometers such as the gratings on board Chandra or XMM-Newton. However, it is not trivial to obtain such data for flares and to date no such diagnostics is available yet.

It is instead well-known that in many flares, both solar and stellar, the temperature peaks and starts to decay well before the emission measure. This is consistently described by models of confined flares: as the heating drops, the temperature begins to decay, while plasma evaporates and makes emission measure increase still for some time.
In our simulations of non-confined flares, instead temperature and emission measure both decay immediately and simultaneously after the heating stops. This difference may indeed be a strong indication of confinement for long-lasting stellar flares (Item 7 in Table 2).

Another significant indication comes from the analysis of the light curves in a wide X-ray passband, such as ASCA/SIS (Item 8 in Table 2). In the case of non-confined flares the light curve invariably drops as soon as the heating stops, with a decay time in the early phase of few seconds (Eq. (11)), and then decays more gradually, with a time scale of few minutes (Eq. (13)). The early decay of the light curve of the confined flare is much more gradual, and becomes even more gradual (hours) if the confining loop is longer than a solar active region loop (Eq. (14)). Unless we hypothesize unlikely and ad hoc heating depositions, e.g. decreasing very gradually and completely driving the decay phase, a sudden drop of the X-ray light 
curve is an expected intrinsic and unavoidable feature of flare events in a non-confined corona.

The light curve in selected X-ray lines are also different when comparing confined and non-confined events (Item 9 in Table 2). In particular, in the model of confined flare the light curves in two lines at different formation temperature are considerably asynchronous: the hotter line peaks and decays earlier. In the non-confined case the two lines evolve practically with the same timing. This diagnostic may become interesting if flare light curves in single X-ray lines are available.

The presence of long-enduring, high speed, expanding plasma fronts may open the opportunity to detect persistent blue-shifted components in high resolution line profiles (Item 10 in Table 2). Upflows of about $400 \mathrm{~km} \mathrm{~s}^{-1}$ have been seen in the Ca XIX and Fe XXV lines in the early thermal phase of some solar flares (e.g. Antonucci et al. 1987, 1990). Hydrodynamic models of confined flares predict that the initial chromospheric evaporation may produce blue-shifts in X-ray lines, but for very short times (less than one minute in active region loops, see Fig. 11). We have shown that a steady broadened component blueshifted by $\sim 300 \mathrm{~km} \mathrm{~s}^{-1}$ may be expected in the FeXXI line at $128.75 \AA$ for the reference non-confined model. This kind of prediction may not provide significant indications when looking at real flare observations for various reasons: blue-shifts are expected only if the flaring region is favourably oriented to the observer; shifts of few hundreds $\mathrm{km} \mathrm{s}^{-1}$ are hardly detectable even with XMM and Chandra high resolution grating spectrometers; although less persistent, significant blue-shifted components may be present even during confined flares in long loops. All these limitations may make any diagnostics of confinement from line shifts rather difficult.

Recent works have pointed the attention to the derivation of the distribution of emission measure with temperature (Item 11 in Table 2) from the analysis of X-ray stellar data (e.g. Drake et al. 2000; Linsky \& Gagne 2001). Non-confined flare models predict a flare emission measure distribution monotonically decreasing with temperature during the heating phase and then losing the highest temperature components and some intermediate temperature components in the decay phase. These distributions are quite different from those typically derived from flaring and non-flaring solar (e.g. Peres et al. 2000; Reale et al. 2001) and non-flaring stellar data, which instead show well-defined $E M$ peaks. This may be a discriminating feature, whenever medium resolution spectra provide reliable emission measure distributions during stellar flares (e.g. Osten et al. 2000).

As a final diagnostic consideration, we point out that, if data show evidence of a flare occurring in a non-confined plasma, then according to our modeling the soft X-ray light curve would faithfully trace the evolution of the heating, an important diagnostics for coronal physics (Item 12 in Table 2). An equivalent information is generally derived from hard X-rays in solar flares (e.g. Golub \& Pasachoff 1997).
We may wonder whether our results, such as the light curves presented in Fig. 7, are affected by the Non Equilibrium of Ionization (NEI) conditions of the plasma. In general, in fact, the X-ray emissivity depends on the thermal history of the emitting plasma parcel, which is unfortunately not straightforward to evaluate. Bocchino et al. (1997) show that the $0.1-2.0 \mathrm{keV}$ X-ray emissivity of an impulsively heated plasma (a situation similar to the rise of the flare in our models) approaches the equilibrium value for ionization time $\log \tau \sim 11 \mathrm{in} \mathrm{s} \mathrm{cm}^{-3}$. This is also true in the ASCA/SIS bandwidth. In the initial phase of the flare the typical density of the bright X-ray emitting plasma is in excess of $10^{9} \mathrm{~cm}^{-3}$, corresponding to an equilibrium time scale of the order of $100 \mathrm{~s}$ : we may expect a modification of the light curve before $100 \mathrm{~s}$, consisting in a higher NEI value reached at the end of the rising phase and a slow descend to the sustained rate. In the fast decay at the end of the heating phase the NEI conditions may have some importance and bring to variations of the light curve in this phase. However, these effects will be reduced for hotter and hotter events, i.e. typical intense stellar flares, because the continuum emission dominates more and more on the line emission. Later on, in the gradual decay, most of the emission comes from the expanding shock, in which we have only a slight decrease of the temperature, and therefore the plasma is only marginally overionized. The small deviation from equilibrium gives quasi-equilibrium conditions during most of the decay phase, thus we do not expect substantial modifications of the global time scales we have worked out.

In summary, there are two basic features that may characterize invariably flares in non-confined regions and may be relatively easy to trace in stellar X-ray data: light curves with very fast decays and synchronous evolution of density and temperature. Both these features are in contrast with evidence from stellar flares. Then we come to an interesting conclusion: the long-lasting X-ray flares typically detected in several stellar observations cannot occur in non-confined atmospheres. This is somewhat unexpected, because long-lasting stellar flares have been typically associated to very large coronal structures (see Sect. 1), which have the non-confined configuration as an asymptotic extreme. This work shows that most likely the long-lasting flares, such as those in active stars, still occur in closed, although large, structures and that the role of confinement of the coronal magnetic field must be invariably significant in those events. In other words, the magnetic fields must always be strong and/or the magnetic structure never breaks open. In the same direction, notice also that non-confined flares appear to involve huge amounts of heating (see Table 1) which may not be entirely realistic when compared to the energy budget of stellar coronae.

If long-duration stellar flares are unlikely to be described as breaking the magnetic confinement, are there any other observed events which instead could be? As mentioned in Sect. 1 long stellar flares are preferentially detected in X-ray observations. However there is a class 
of coronal variations which occur on small time scales and which have been detected on dMe star UV Ceti by ROSAT (Schmitt et al. 1993). The possibility that such short events may be interpreted as small but very intense non-confined events should be explored.

This study in some way shows an interesting theoretical perspective to be compared with SNR models and Coronal Mass Ejections (CME) models. Our model shows an overheated plasma which expands under the effect of heating in a hot and thermally conducting stratified atmosphere, and we provide for it characteristic scalings of general validity (Eqs. (10), (11), (13)). For instance, since the time scale of the late decay $\tau_{\mathrm{f}}$ (Eq. (13)) depends practically only on the speed of the evaporation front and on the thermal plasma conditions far from the flaring region, it is little dependent on the flare heating and on other model parameters: it is a general decay time scale for shell fronts expanding in a stratified conducting corona. It should be noted that application to other astrophysical systems is not trivial. In SNR models, plasma expands in a much cooler, thinner and less conducting medium. On the other hand, CME's are mostly observed in the UV band, and are therefore to be modeled as relatively cool perturbations, whereas the non-confined fronts modeled here are at standard coronal temperatures. For the latter fronts to become proper CME's, a mechanism to thermally insulate the fronts from the surrounding corona should be invoked, so that they are free to cool by radiation and to emit in the UV band (e.g. Ciaravella et al. 2001).

\section{Conclusions}

Stellar flares are generally observed to evolve and decay on time scales ranging from several hours to days, suggesting large flaring regions or even lack of magnetic confinement. This work shows that the hydrodynamic evolution of flares occurring in non-confined atmospheres leads invariably to a much faster decay of the brightness (on time scales of very few minutes) after the heating phase, in a wide range of the physical parameters. One of the main implications is that the long duration of stellar flares is not indicative of non-confined plasma. Reversing the argument, it is highly probable that the observed long-lasting stellar X-ray flares involve forms of plasma confinement in closed coronal structures. We have pointed out other characteristic features, with more or less diagnostical power, e.g. the synchronous evolution of density and temperature, summarized in Table 2, that seem to indicate that X-ray observed stellar flares involve mostly plasma confined in closed structures.

Although this work simulates rather extreme conditions of flares occurring in completely non-confined atmospheres, the result that long-lasting stellar flares are likely not occurring in large open structures largely motivates its validity.

Furthermore this work predicts the possible existence of a new phenomenological class of events, characterized by peculiar light curves with a limited range of time scales.
Such kind of events may be addressed by observations at high sensitivity levels, which allow for high time resolution and high signal-to-noise ratio, such as those obtained from the current missions Chandra and XMM-Newton.

Acknowledgements. This work was supported in part by Agenzia Spaziale Italiana and by Ministero della Università e della Ricerca Scientifica e Tecnologica. Simulations have been executed on the Cray T3E at CINECA (Bologna, Italy), on a Compaq server and on a Compaq HPC Cluster at Osservatorio Astronomico di Palermo (Italy).

\section{References}

Antonucci, E., Dodero, M. A., Peres, G., Serio, S., \& Rosner, R. 1987, ApJ, 322, 522

Antonucci, E., Dodero, M. A., \& Martin, R. 1990, ApJS, 73, 137

Arnaud, M., \& Raymond, J. 1992, ApJ, 398, 394

Bedogni, R., \& Woodward, P. R. 1990, A\&A, 231, 481

Bocchino, F., Maggio, A., \& Sciortino, S. 1997, ApJ, 481, 872

Cheng, C.-C., Oran, E. S., Doschek, G. A., Boris, J. P., \& Mariska, J. T. 1983, ApJ, 265, 1090

Ciaravella, A., Raymond, J. C., Reale, F., Strachan, L., \& Peres, G. 2001, ApJ, 557, 351

Dere, K. P., Landi, E., Mason, H. E., et al. 1997, A\&AS, 125, 149

Drake, J. J., Peres, G., Orlando, S., Laming, J. M., \& Maggio, A. 2000, ApJ, 545, 1074

Favata, F. 2001, Proc. Xray-Astronomy 2000, Mondello (Palermo), Sept. 4-9, 2000, ed. R. Giacconi, L. Stella, \& $\mathrm{S}$. Serio, in press

Favata, F., Micela, G., Reale, F., Sciortino, S., \& Schmitt, J. H. M. M. 2000, A\&A, 362, 628

Favata, F., \& Schmitt, J. H. M. M. 1999, A\&A, 350, 900

Golub, L., \& Pasachoff, J. M. 1997, The solar corona (Cambridge Univ. Press, Cambridge)

Graffagnino, V. G., Wonnacott, D., \& Schaeidt, S. 1995, MNRAS, 275, 129

Grevesse, N., \& Anders, E. 1991, in Solar Interior and Atmosphere, ed. A. N. Cox, W. C. Livingston, \& M. S. Matthews (Tucson: Univ. Arizona Press), 1227

Haisch, B. M. 1983, in Activity in red-dwarf stars, ed. P. B. Byrne, \& M. Rodonò, Proceedings of the Seventy-first Colloquium, Catania, Italy, August 10-13, 1982 (A83-47476 23-90) (Dordrecht, D. Reidel)

Jakimiec, J., Sylwester, B., Sylwester, J., et al. 1992, A\&A, 253, 269

Linsky, J. L. 1981, in Solar Phenomena in Stars and Stellar Systems, ed. R. M. Bonnet, \& A. K. Dupree (Dordrecht: Reidel), 99

Linsky, J. L., \& Gagne, M. 2001, A\&AS, 198, L4405

Maggio, A., Pallavicini, R., Reale, F., \& Tagliaferri, G. 2000, A\&A, 356, 627

Maggio, A., \& Peres, G. 1997, A\&A, 325, 237

McClymont, A. N., \& Canfield, R. C. 1983, ApJ, 265, 483

Mutel, R. L., Lestrade, J. F., Preston, R. A., \& Phillips, R. B. 1985, ApJ, 289, 262

Nagai, F. 1980, Sol. Phys., 68, 351

Osten, R. A., Brown, A., Ayres, T. R., et al. 2000, ApJ, 544, 953

Peres, G., Reale, F., Serio, S., \& Pallavicini, R. 1987, ApJ, 312, 895 
Peres, G., Rosner, R., Serio, S., Vaiana, G. S. 1982, ApJ, 252, 791

Raymond, J. C., \& Smith, B. W. 1977, ApJS, 35, 419

Reale, F. 1995, Comp. Phys. Comm., 86, 13

Reale, F. 2001, Proc of 35-th ESLAB Symposium Stellar Coronae in the Chandra and XMM-Newton Era, ESTEC, 25-29 June 2001, ed. F. Favata, \& J. Drake, in press

Reale, F., Betta, R., Peres, G., Serio, S., \& McTiernan, J. 1997, A\&A, 325, 782

Reale, F., Peres, G., Serio, S., Rosner, R., \& Schmitt, J. H. M. M. 1988, ApJ, 328, 256

Reale, F., Peres, G., \& Serio, S. 1990, Nuovo Cimento B, 105, 1235

Reale, F., Peres, G., Serio, S., et al. 2000, ApJ, 535, 423

Rosenau, P., \& Frankenthal, S. 1976, ApJ, 208, 633
Rosner, R., Tucker, W., \& Vaiana, G. 1978, ApJ, 220, 643

Schmitt, J. H. M. M., Collura, A., Sciortino, S., et al. 1990, ApJ, 365, 704

Schmitt, J. H. M. M., Haisch, B., \& Barwig, H. 1993, ApJL, 419, L81

Serio, S., Reale, F., Jakimiec, J., Sylwester, B., \& Sylwester, J. 1991, A\&A, 241, 197

Sylwester, B., Sylwester, J., Serio, S., et al. 1993, A\&A, 267, 586

Tsuboi, Y., Imanishi, K., Koyama, K., Grosso, N., \& Montmerle, T. 2000, ApJ, 532, 1089

van den Oord, G. H. J., \& Mewe, R. 1989, A\&A, 213, 245

van den Oord, G. H. J., Mewe, R., \& Brinkman, A. C. 1988, A\&A, 205, 181

White, S. M., \& Franciosini, E. 1995, ApJ, 444, 342 